\title{
FAST COMPUTATION OF FOURIER INTEGRAL OPERATORS*
}

\author{
EMMANUEL CANDÈS ${ }^{\dagger}$, LAURENT DEMANET $^{\ddagger}$, AND LEXING YING ${ }^{\S}$
}

\begin{abstract}
We introduce a general purpose algorithm for rapidly computing certain types of oscillatory integrals which frequently arise in problems connected to wave propagation, general hyperbolic equations, and curvilinear tomography. The problem is to numerically evaluate a so-called Fourier integral operator (FIO) of the form $\int e^{2 \pi i \Phi(x, \xi)} a(x, \xi) \hat{f}(\xi) \mathrm{d} \xi$ at points given on a Cartesian grid. Here, $\xi$ is a frequency variable, $\hat{f}(\xi)$ is the Fourier transform of the input $f, a(x, \xi)$ is an amplitude, and $\Phi(x, \xi)$ is a phase function, which is typically as large as $|\xi|$; hence the integral is highly oscillatory. Because a FIO is a dense matrix, a naive matrix vector product with an input given on a Cartesian grid of size $N$ by $N$ would require $O\left(N^{4}\right)$ operations. This paper develops a new numerical algorithm which requires $O\left(N^{2.5} \log N\right)$ operations and as low as $O(\sqrt{N})$ in storage space (the constants in front of these estimates are small). It operates by localizing the integral over polar wedges with small angular aperture in the frequency plane. On each wedge, the algorithm factorizes the kernel $e^{2 \pi i \Phi(x, \xi)} a(x, \xi)$ into two components: (1) a diffeomorphism which is handled by means of a nonuniform FFT and (2) a residual factor which is handled by numerical separation of the spatial and frequency variables. The key to the complexity and accuracy estimates is the fact that the separation rank of the residual kernel is provably independent of the problem size. Several numerical examples demonstrate the numerical accuracy and low computational complexity of the proposed methodology. We also discuss the potential of our ideas for various applications such as reflection seismology.
\end{abstract}

Key words. Fourier integral operators, generalized Radon transform, separated representation, nonuniform fast Fourier transform, matrix approximation, operator compression, randomized algorithms, reflection seismology

AMS subject classifications. 35S30, 65F $30,86 \mathrm{~A} 15$

DOI. $10.1137 / 060671139$

1. Introduction. This paper introduces a general purpose algorithm to compute the action of linear operators which are frequently encountered in analysis and scientific computing. These operators take the form

$$
(L f)(x)=\int_{\mathbb{R}^{d}} a(x, \xi) e^{2 \pi i \Phi(x, \xi)} \hat{f}(\xi) \mathrm{d} \xi,
$$

where $\Phi(x, \xi)$ is a phase function that is smooth in $(x, \xi)$ for $\xi \neq 0$ and obeys the homogeneity relation $\Phi(x, \lambda \xi)=\lambda \Phi(x, \xi)$ for $\lambda$ positive, and $a(x, \xi)$ is a smooth amplitude term. As is standard, $\hat{f}$ is the Fourier transform of $f$ defined by

$$
\hat{f}(\xi)=\int_{\mathbb{R}^{d}} f(x) e^{-2 \pi i x \xi} \mathrm{d} x .
$$

*Received by the editors September 30, 2006; accepted for publication (in revised form) April 9, 2007; published electronically October 17, 2007. This work was partially supported by an NSF grant CCF-0515362 and a DOE grant DE-FG03-02ER25529.

http://www.siam.org/journals/sisc/29-6/67113.html

${ }^{\dagger}$ Applied and Computational Mathematics, California Institute of Technology, Pasadena, CA 91125 (ecandes@acm.caltech.edu).

${ }^{\ddagger}$ Department of Mathematics, Stanford University, Stanford, CA 94305 (laurent@math.stanford. edu).

$\S$ Department of Mathematics, University of Texas at Austin, Austin, TX 78712 (lexing@ math.utexas.edu). 
With the proper regularity assumptions on the amplitude and the phase ${ }^{1}(1.1)$ defines a class of oscillatory integrals known as Fourier integral operators (FIOs). FIOs are the subject of considerable study for many of the operators encountered in physics and other fields. For instance, most differential and pseudodifferential operators are FIOs. Convolutions and multiplications by smooth functions are FIOs. Some "principal value" integrals are FIOs. And the list goes on.

An especially important example of a FIO is the solution operator to the freespace wave equation in $\mathbb{R}^{d}, d>1$,

$$
\frac{\partial^{2} u}{\partial t^{2}}(x, t)=c^{2} \Delta u(x, t),
$$

with initial conditions $u(x, 0)=u_{0}(x)$ and $\frac{\partial u}{\partial t}(x, 0)=0$, say. Everyone knows that for constant speeds, the Fourier transform decouples the different frequency components of $u$. Each Fourier component obeys an ordinary differential equation which can be solved explicitly. The solution $u(x, t)$ is the superposition of these Fourier modes and is given by

$$
u(x, t)=\frac{1}{2}\left(\int e^{2 \pi i(x \cdot \xi+c|\xi| t)} \hat{u_{0}}(\xi) \mathrm{d} \xi+\int e^{2 \pi i(x \cdot \xi-c|\xi| t)} \hat{u_{0}}(\xi) \mathrm{d} \xi\right) .
$$

The connection is now clear: the solution operator is the sum of two FIOs with phase functions

$$
\Phi_{ \pm}(x, \xi)=x \cdot \xi \pm c|\xi| t
$$

For variable but reasonably smooth sound speeds $c(x)$, the solution operator is for small times a sum of two FIOs with more complicated phases and amplitudes. In particular, the phase can be constructed from the optical traveltime in a medium with index of refraction $1 / c(x)$; see [11] for details.

An important property of FIOs is that they displace wavefront sets and singular supports (where the solution is singular), which is the favored mathematical way of formulating propagation of singularities along characteristic manifolds for hyperbolic equations [20]. For this reason, the solution operators for heat equations or Schrödinger equations are not FIOs, because these equations diffuse or disperse wavefronts. More precisely, their solution operators could potentially be put in the form (1.1), but the homogeneity relation $\Phi(x, \lambda \xi)=\lambda \Phi(x, \xi)$ would be lost.

In short, it is useful to think of FIOs as proxies for the solution operator to large classes of hyperbolic differential equations.

1.1. FIO computations. Numerical simulation of free wave propagation with constant sound speed is straightforward. As long as the solution $u(x, t)$ is sufficiently well localized both in space and frequency, it can be computed accurately and rapidly by applying the sequence of steps below.

1. Compute the fast Fourier transform (FFT) of $u_{0}$.

2. Multiply the result by $e^{ \pm 2 \pi i c|\xi| t}$, and sum as in (1.4).

\footnotetext{
${ }^{1}$ The amplitude should be $C^{\infty}$ and obey (2.3). The phase should be $C^{\infty}$ except at $\xi=0$. In general, we do not require the nondegeneracy condition $\operatorname{det}\left(\frac{\partial^{2} \Phi}{\partial x_{i} \partial \xi_{j}}\right) \neq 0$ if the only goal is to apply the FIO (note that such a FIO may not be invertible). With this being said, we can handle only canonical relations that are locally the graph of a function; i.e., we do not deal with multivalued phases.
} 
3. Compute the inverse FFT.

Of course, this works only in the very special case where the amplitude $a$ is independent of $x$, and where the phase is of the form $x \cdot \xi$ plus a function of $\xi$ alone. Expressed differently, this works when the FIO is shift-invariant so that it is diagonal in the Fourier basis. Note that there is in general no formula for the eigenfunctions when $\Phi$ or $a$ depend on $x$. Computing these eigenfunctions on the fly is out of the question when the objective is merely to compute the action of the operator. (Note that even if the spectral decomposition of the operator were available, it is not clear how one would use it to speed up computations.)

The object of this paper is to find an algorithm that is considerably faster than evaluating (1.1) by direct quadratures and is yet suited to handle large classes of phases and amplitudes. Most of the existing fast summation techniques rely on either the nonoscillatory behavior (such as wavelet-based techniques [8]) or the existence of a low rank approximation (fast multipole methods [26], hierarchical matrices [27], pseudodifferential separation [4]). The difficulty here is that the kernel $e^{2 \pi i \Phi(x, \xi)}$ is highly oscillatory and does not have a low rank separated approximation. Therefore, all the modern techniques are not directly applicable.

The main claim of this paper, however, is that there is a way to decompose the operator into a sum of components for which the oscillations are well understood and low rank representations are available. In addition, the number of such components is reasonably small, which paves the way to faster algorithms. Before expanding on this idea, we first explain the discretization of the operator (1.1).

1.2. Discretization. For simplicity, we restrict our attention in this paper to the two-dimensional case $d=2$. The main ideas apply readily to the higher dimensions, though the analysis and implementation would be more involved.

Just as the discrete Fourier transform is the digital analogue of the continuous Fourier transform, one can also introduce discrete FIOs. Given a function $f$ defined on a Cartesian grid $X=\left\{x=\left(\frac{n_{1}}{N}, \frac{n_{2}}{N}\right), 0 \leq n_{1}, n_{2}<N\right.$, and $\left.n_{1}, n_{2} \in \mathbb{Z}\right\}$, we simply define the discrete FIO by

$$
(L f)(x):=\frac{1}{N} \sum_{\xi \in \Omega} a(x, \xi) e^{2 \pi i \Phi(x, \xi)} \hat{f}(\xi)
$$

for every $x \in X$. (We are sorry for overloading the symbol $L$ to denote both the discrete and continuous objects but there will be no confusion in what follows.) The summation above is taken over all $\Omega=\left\{\xi=\left(n_{1}, n_{2}\right),-\frac{N}{2} \leq n_{1}, n_{2}<\frac{N}{2}\right.$, and $n_{1}, n_{2} \in$ $\mathbb{Z}\}$, and throughout this paper, we will assume that $N$ is an even integer. Here and below, $\hat{f}$ is the discrete Fourier transform (DFT) of $f$ and is defined as

$$
\hat{f}(\xi)=\frac{1}{N} \sum_{x \in X} e^{-2 \pi i x \cdot \xi} f(x)
$$

The normalizing constant $\frac{1}{N}$ in (1.5) (resp., (1.6)) ensures that $L$ (resp., the DFT) is a discrete isometry in the case where $\Phi(x, \xi)=x \cdot \xi$.

The formula (1.5) turns out to be an accurate discretization of (1.1) as soon as $f$ obeys standard localization estimates both in space and frequency. A justification of this fact, however, would go beyond the scope of this paper and is omitted. In the remainder of the paper, we will take (1.5) as the quantity we wish to compute once we are given a phase and an amplitude function. 
The parameter $N$ measures the size and difficulty of the computational problem. In a nutshell, it corresponds to the number of points which are needed in each direction to accurately sample the continuous object $f(x)$. This is the reason why $N$ will be a central quantity throughout the rest of paper.

As mentioned earlier, the straightforward method for computing (1.5) simply evaluates the summation independently for each $x$. Since each sum takes $O\left(N^{2}\right)$ operations and there are $N^{2}$ grid points in $X$, this strategy requires $O\left(N^{4}\right)$ operations. When $N$ is moderately large, this can be prohibitive. This paper describes a novel algorithm which computes all the values of $L f(x)$ for $x \in X$ with high accuracy in $O\left(N^{2.5} \log N\right)$ operations. The only requirement is that the amplitude and the phase obey mild smoothness conditions, which are in fact standard. As a matter of fact, our algorithm can be applied even to the cases where the nondegeneracy condition $\operatorname{det}\left(\frac{\partial^{2} \Phi}{\partial x_{i} \partial \xi_{j}}\right) \neq 0$ does not hold.

1.3. Separation within angular wedges. This section outlines the main idea of the paper. Let $\arg \xi$ be the angle between $\xi$ and the horizontal vector $(1,0)$, and partition the frequency domain into a family of angular wedges $\left\{W_{\ell}\right\}$ defined by

$$
W_{\ell}=\{\xi:(2 \ell-1) \pi / \sqrt{N} \leq \arg \xi<(2 \ell+1) \pi / \sqrt{N}\}
$$

for $0 \leq \ell<\sqrt{N}$ (assume $\sqrt{N}$ is an integer). An important property of these wedges is that each $W_{\ell}$ satisfies the parabolic relationship

$$
\text { length } \simeq \text { width } h^{2}
$$

up to multiplicative constants independent of $N$. There are $O(\sqrt{N})$ such wedges, as illustrated in Figure 1.

For each wedge $W_{\ell}$, we let $\chi_{\ell}$ be the indicator function of $W_{\ell}$. Similarly, we denote by $\hat{\xi}_{\ell}$ the unit vector pointing to the center direction of $W_{\ell}$

$$
\hat{\xi}_{\ell}=\left(\cos \frac{2 \ell \pi}{\sqrt{N}}, \sin \frac{2 \ell \pi}{\sqrt{N}}\right) .
$$

It follows from the identify $\sum_{\ell} \chi_{\ell}(\xi)=1$ that one can decompose the operator $L$ as $\sum_{\ell} L_{\ell}$, where

$$
\left(L_{\ell} f\right)(x)=\frac{1}{N} \sum_{\xi} a(x, \xi) e^{2 \pi i \Phi(x, \xi)} \chi_{\ell}(\xi) \hat{f}(\xi)
$$

Within each wedge $W_{\ell}$, we can perform a Taylor expansion of $\Phi(x, \xi)$ in the second variable, around the point $\hat{\xi}_{\ell}|\xi|$. There is a point $\xi^{\star}$ which belongs to the line segment $\left[\hat{\xi}_{\ell}|\xi|, \xi\right]$ such that

$\Phi(x, \xi)=\Phi\left(x, \hat{\xi}_{\ell}|\xi|\right)+\nabla_{\xi} \Phi\left(x, \hat{\xi}_{\ell}|\xi|\right) \cdot\left(\xi-\hat{\xi}_{\ell}|\xi|\right)+\frac{1}{2}\left(\xi-\hat{\xi}_{\ell}|\xi|\right)^{T} \nabla_{\xi \xi} \Phi\left(x, \xi^{\star}\right)\left(\xi-\hat{\xi}_{\ell}|\xi|\right)$.

By homogeneity of the phase $(\Phi(x, \lambda \xi)=\lambda \Phi(x, \xi)$ for $\lambda>0)$, it holds that $\Phi(x, \xi)=$ $\xi \cdot \nabla_{\xi} \Phi(x, \xi)$ and $\nabla_{\xi} \Phi(x, \xi)=\nabla_{\xi} \Phi(x, \hat{\xi})$. The first and third terms in the above expression cancel, and thus

$$
\Phi(x, \xi)=\nabla_{\xi} \Phi\left(x, \hat{\xi}_{\ell}\right) \cdot \xi+\frac{1}{2}\left(\xi-\hat{\xi}_{\ell}|\xi|\right)^{T} \nabla_{\xi \xi} \Phi\left(x, \xi^{\star}\right)\left(\xi-\hat{\xi}_{\ell}|\xi|\right) .
$$




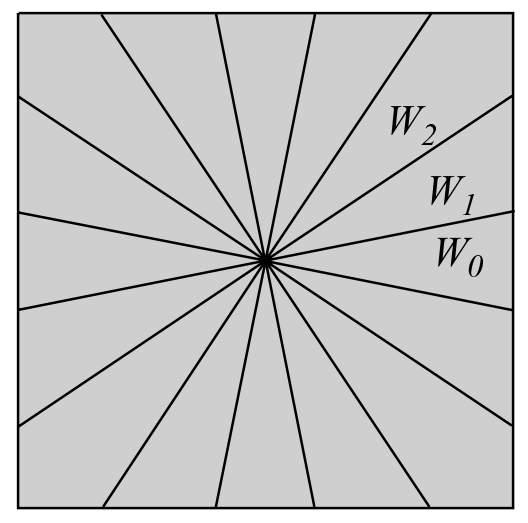

FIG. 1. The frequency domain is partitioned into $\sqrt{N}$ equiangular wedges.

The first term $\nabla_{\xi} \Phi\left(x, \hat{\xi}_{\ell}\right) \cdot \xi$, which is linear in $\xi$, is called the linearized phase and poses no problem as we will see later on. The rest, denoted as $\Phi_{\ell}(x, \xi)=\Phi(x, \xi)-$ $\nabla_{\xi} \Phi\left(x, \hat{\xi}_{\ell}\right) \cdot \xi$ and called the residual phase, is of order $O(1)$ for $\xi \in W_{\ell}$, independently of $N$. This follows from

$$
\nabla_{\xi \xi} \Phi\left(x, \xi^{\star}\right)=O\left(\left|\xi^{\star}\right|^{-1}\right)=O\left(|\xi|^{-1}\right),
$$

since $\Phi(x, \xi)$ is homogeneous of degree 1 in $\xi$, together with

$$
\left.\left|\xi-\hat{\xi}_{\ell}\right| \xi\right|^{2}=O\left(|\xi|^{2} / N\right)=O(|\xi|)
$$

for all $|\xi| \leq N$, which uses the fact that the shape of $W_{\ell}$ obeys the parabolic relationship (1.7).

Because the residual phase $\Phi_{\ell}(x, \xi)$ is of order $O(1)$ independently of $N$, we say that the function $e^{2 \pi i \Phi_{\ell}(x, \xi)}$ is nonoscillatory. Under mild assumptions, this observation guarantees the existence of a low rank separated representation which decouples the variables $x$ and $\xi$ and approximates the complex exponential very well. Define the $\epsilon$-separation rank of a function $f(x, y)$ of two variables as the smallest integer $r_{\epsilon}$ for which there exist $c_{n}(x), d_{n}(y)$ such that

$$
\left|f(x, y)-\sum_{n=0}^{r_{\epsilon}-1} c_{n}(x) d_{n}(y)\right| \leq \epsilon .
$$

Then we prove the following theorem in section 2 .

TheOREm. For all $0<\epsilon \leq 1$, there exist $N^{*}>0$ and $C>0$ such that for all $N \geq N^{*}$, the $\epsilon$-separation rank of $e^{2 \pi i \Phi_{\ell}(x, \xi)}$ for $x \in[0,1]^{2}$ and $\xi \in W_{\ell}$ obeys

$$
r_{\epsilon} \leq \log _{2}\left(C \epsilon^{-1}\right) .
$$

In section 2 we make explicit the values of the constants $N^{*}$ and $C$ by relating them, among other things, to the smoothness of $\Phi$ and the angular span of $W_{\ell}$. We will also provide results in the case where $N \leq N^{*}$ and explain why the separation rank for the amplitude is also under control.

The point of the theorem is that the bound on the $\epsilon$-rank does not grow as a function of $N$-in fact, the threshold condition on $N$ indicates that the $\epsilon$-rank decays 
as $N$ grows. The logarithmic dependence on $\epsilon$ is the signature of what is usually called spectral accuracy.

Note that the decomposition into frequency wedges obeying the parabolic scaling has a long history in mathematics. A multiscale version of this partitioning, the second dyadic decomposition, was introduced by Fefferman in 1973 for the study of Bochner-Riesz multipliers [22] and used by Seeger, Sogge, and Stein in 1991 to prove a sharp $L^{p}$-boundedness result for FIOs [36]. More recently, it also served as the basis for the construction of curvelets, with applications to sparsity of FIOs and related results for wave equations $[37,10,11]$.

1.4. Outline of the algorithm. The low rank separated representation provided by the theorem above offers us a way to compute (1.5) efficiently with high accuracy. Each term in the decomposition $L f=\sum_{\ell} L_{\ell} f$ can be further simplified as follows:

$$
\begin{aligned}
\left(L_{\ell} f\right)(x) & =\frac{1}{N} \sum_{\xi} a(x, \xi) e^{2 \pi i \Phi(x, \xi)} \chi_{\ell}(\xi) \hat{f}(\xi) \\
& =\frac{1}{N} \sum_{\xi} e^{2 \pi i \nabla_{\xi} \Phi\left(x, \hat{\xi}_{\ell}\right) \cdot \xi} a(x, \xi) e^{2 \pi i \Phi_{\ell}(x, \xi)} \chi_{\ell}(\xi) \hat{f}(\xi) \\
& =\frac{1}{N} \sum_{\xi} e^{2 \pi i \nabla_{\xi} \Phi\left(x, \hat{\xi}_{\ell}\right) \cdot \xi} \sum_{t=1}^{\infty} \gamma_{\ell t}^{x}(x) \gamma_{\ell t}^{\xi}(\xi) \chi_{\ell}(\xi) \hat{f}(\xi) \\
& =\frac{1}{N} \sum_{t=1}^{\infty} \gamma_{\ell t}^{x}(x) \sum_{\xi} e^{2 \pi i \nabla_{\xi} \Phi\left(x, \hat{\xi}_{\ell}\right) \cdot \xi}\left[\gamma_{\ell t}^{\xi}(\xi) \chi_{\ell}(\xi) \hat{f}(\xi)\right] .
\end{aligned}
$$

Our analysis guarantees that the sum over $t$ can be truncated to a fixed, hopefully small number of terms without significant loss of precision.

In order to carry out the final summation over $t$, we first need to construct the functions $\gamma_{\ell t}^{x}(x)$ and $\gamma_{\ell t}^{\xi}(\xi)$. Sections 3.1 and 3.2 discuss two different methods of finding these functions. In section 3.1 we present an elementary deterministic approach, while in section 3.2 we present a randomized approach that offers better efficiency both timewise and storagewise. Assuming that $\gamma_{\ell t}^{x}(x)$ and $\gamma_{\ell t}^{\xi}(\xi)$ are available for all values of $\ell$ and $t$, the computation of $(L f)$ for a given $f$ consists of the following four steps:

1. Fourier transform $f$ by means of the FFT to get $\hat{f}$.

2. Choose a bound $q$ greater than the $\varepsilon$-rank $r_{\varepsilon}$. For each $\ell$ and $t \leq q$, form $\hat{f}_{\ell t}(\xi):=\gamma_{\ell t}^{\xi}(\xi) \chi_{\ell}(\xi) \hat{f}(\xi)$.

3. For each $\ell$ and $t \leq q$, compute $g_{\ell t}(x):=\sum_{\xi} e^{2 \pi i \nabla_{\xi} \Phi\left(x, \hat{\xi}_{\ell}\right) \cdot \xi} \hat{f}_{\ell t}(\xi)$ by means of a nonuniform FFT algorithm.

4. Compute $(L f)(x) \approx \frac{1}{N} \sum_{\ell} \sum_{t=1}^{q} \gamma_{\ell t}^{x}(x) g_{\ell t}(x)$.

The only step that requires further discussion is the computation of $g_{\ell, t}$. We defer the details to section 3.4.

It is instructive to understand why linearizing the phase is so important. If we disregard the error introduced by the discretization in $\xi$, we observe that $g_{\ell, t}(x)$ is simply

$$
g_{\ell, t}(x)=f_{\ell, t}\left(\nabla_{\xi} \Phi\left(x, \hat{\xi}_{\ell}\right)\right)
$$

Copyright (c) by SIAM. Unauthorized reproduction of this article is prohibited. 
The interpretation of an oscillatory integral in the Fourier domain as a diffeomorphism is possible only when the phase is linear in $\xi$. For each $\ell$ and $t$, the computation of $g_{\ell, t}$, which is an interpolation problem, is therefore a much simpler problem than applying the original operator. Admittedly, diffeomorphisms do not provide accurate approximations to FIOs over angular wedges, but the content of our analysis in section 2 shows that the computational budget to make up for the residual is safely under control.

1.5. Significance. Applying nontrivial FIOs repeatedly has proved to be the computational bottleneck in various inverse problems. There is serious scientific as well as industrial interest in speeding up FIO computations, and accordingly many resources have been invested over the past decades in engineering better codes.

We believe that the ideas introduced in this paper provide new directions. To explain and illustrate this contrast, let us consider an important example from the field of reflection seismology: Kirchhoff migration. The problem is to produce an image of the discontinuities in the Earth's upper crust from seismograms, i.e., wave measurements $f_{s}\left(t, x_{r}\right)$ parameterized by time $t$, receiver coordinate $x_{r}$, and source coordinate $x_{s}$. The core of Kirchhoff migration is a generalized Radon transform (GRT) which, (in its shot-gather version) consists in integrating several different functions $f_{s}\left(t, x_{r}\right)$ indexed by $s$ over a fixed set of curves, determined as the level lines of a traveltime function $\tau\left(x, x_{r}\right)+\tau\left(x, x_{s}\right)$, and modulated by an amplitude $a\left(t, x_{r}, x_{s}\right)$ :

$$
g_{s}(x)=\int \delta\left(t-\tau\left(x, x_{r}\right)-\tau\left(x, x_{s}\right)\right) a\left(t, x_{r}, x_{s}\right) f_{s}\left(t, x_{r}\right) \mathrm{d} t \mathrm{~d} x_{r} .
$$

The functions $g_{s}(x)$ determine the model. A stack operation over the $s$ index then allows us to recover the adequate physical parameters of the Earth, like the speed of sound. When $x_{r}$ is one-dimensional, it is useful to think of the traveltime level curves as distorted hyperbolas in the $\left(t, x_{r}\right)$ plane. A standard notation for Kirchhoff migration is $g_{s}(x)=\left(F^{*} f_{s}\right)(x)$, where $F^{*}$ is called the imaging operator.

Equation (1.10) is in fact a backprojection strategy for approximately inverting a forward GRT $-F$ in the above notation - which in turn comes from a linearization for the physical parameters about small perturbations, combined with a leading-order high-frequency approximation of the wave equation's Green's function. This view of migration as a GRT is now authoritative in the field of seismic imaging and was pioneered in work by Beylkin and also Miller, Tarantola, Lailly, and Rakesh. The original papers by Beylkin and collaborators are [6, 7, 33]. For more references, see the review article [39] and references therein.

A GRT like (1.10), with a proper cutoff amplitude, can be put in a FIO form suitable for our algorithm. For convenience, the appendix explains why integration along ellipses - a simple GRT - is a sum of two FIOs.

The standard algorithm for applying the imaging operator as in (1.10) is a simple quadrature of $f\left(t, x_{r}\right)$, interpolated and integrated along each curve $t=\tau\left(x, x_{r}\right)+$ $\tau\left(x, x_{s}\right)$ (parameterized by $x$ ). Assume again that $x_{r}$ is one-dimensional for simplicity. If the data $f\left(t, x_{r}\right)$ oscillates at a wavelength comparable to the grid spacing $1 / N$, then an accurate quadrature on a smooth curve requires $O(N)$ points. Since $x$ takes on $O\left(N^{2}\right)$ values, the curve integration results in a total complexity of $O\left(N^{3}\right)$ for applying the imaging operator (which is of course better than the $O\left(N^{4}\right)$ complexity of the naive summation).

The first claim of this paper is that a potentially more attractive computational strategy for computing (1.10) is to transform it into FIOs, by considering the data 
$f_{s}\left(t, x_{r}\right)$ in the frequency domain (in both variables $t$ and $x_{r}$ ). The asymptotic complexity is then reduced to $O\left(N^{2.5} \log N\right)$ for the algorithm presented in this paper.

The second claim is that since our algorithm is based on FIOs and not just GRTs, it can potentially handle more general migration and imaging operators. For instance, the true imaging operator $F^{*}$ is almost never a GRT like (1.10). If more terms are kept in the geometric optics approximation leading to (1.10), then the resulting migration operator is no longer a GRT, although it is still a FIO under some very general assumptions; see [38] for a detailed exposition. This observation is akin to the fact that the retarded propagator of the wave equation in two dimensions is not a distribution strictly supported on the boundary of the light cone - only its singular support coincides with the boundary of the cone.

The advantages of the proposed algorithm should now be clear: quite general FIOs can be handled with an asymptotic computational complexity which is lower than that required for GRT summation, i.e., $O\left(N^{2.5} \log N\right)$ versus $O\left(N^{3}\right)$, and this without making any curvilinear approximation. In addition, we will show that the storage overhead (on top of storing the phase and amplitude) is negligible and scales like $O(\sqrt{N})$.

We have discussed only applications to reflection seismology, but there are many other areas where nontrivial FIOs are computed routinely, e.g., as part of solving an inverse problem. Examples in radar imaging, ultrasound imaging, and electron microscopy all come to mind. Some Hough transforms for feature detection in image processing can also be formulated as FIOs. In short, the ideas presented in this paper may enable the speedup of fundamental computations in a variety of problem areas.

1.6. Related work. In the case where $\Phi(x, \xi)=x \cdot \xi$, the operator is said to be pseudodifferential. In this simpler setting, it is known that separated variable expansions of the symbol $a(x, \xi)$ are good strategies for reducing complexity. For instance, Bao and Symes [4] propose an $O\left(N^{2} \log N\right)$ numerical method based on a Fourier series expansion of the symbol in the angular variable $\arg \xi$, and a polyhomogeneous expansion in $|\xi|$, which is a particularly effective example of separation of variables.

Another popular approach for compressing operators is to decompose them in a well-chosen, possibly adaptive basis of $L^{2}$. Once a sparse representation is achieved, evaluation simply consists of applying a sparse matrix in the transformed domain. In the case of one-dimensional oscillatory integrals, this program was advocated and carried out by Bradie, Coifman, and Grossman [9] and Averbuch et al. [3]. In spite of these successes, the generalization to multiple dimensions has so far remained an open problem. We will come back to this question in section 5 and in particular will discuss the relationship with modern multiscale transformations such as curvelets $[10,11]$ and wave atoms $[15,16]$.

We would also like to acknowledge the line of research related to Filon-type quadratures for oscillatory integrals [29]. When the integrand is of the form $g(x) e^{i k x}$ with $g$ smooth and $k$ large, it is not always necessary to sample the integrand at the Nyquist rate. For instance, integration of a polynomial interpolant of $g$ (Filon quadrature) provides an accurate approximation to $\int g(x) e^{i k x} \mathrm{~d} x$ using fewer and fewer evaluations of the function $g$ as $k \rightarrow \infty$. While these ideas are important, they are not directly applicable in the case of FIOs. The reasons are threefold. First, we make no notable assumption on the support of the function to which the operator is applied, meaning that the oscillations of $\hat{f}(\xi)$ may be on the same scale as those of the exponential $e^{2 \pi i \Phi(x, \xi)}$. Second, the phase does not in general have a simple formula that would lend itself to precomputations. And third, Filon-type quadratures do not 
address the problem of simplifying computations of several such oscillatory integrals at once (i.e., computing a family of integrals indexed by $x$ in the case of FIOs).

Finally, we remark that FIOs are also interesting when the canonical relation is nontrivial - that is, multivalued phase - because they allow us to study propagation of singularities of hyperbolic equations in regimes of multipathing and caustics $[28,20]$. To mathematicians taking this specialized viewpoint, the focus of the present paper may appear restrictive. Our outlook and ambition are different. We find FIOs to be interesting mathematical objects even when the canonical relation is a graph and degenerates to the gradient of a phase. Our concern is to understand their structure from an operational standpoint and exploit it to design efficient numerical algorithms. In fact, we expect this paper to be the first of a projected series which will eventually deal with more complex setups.

1.7. Contents. The rest of the paper is organized as follows. Section 2 proves all the analytical estimates which support our methodology. In section 3, we describe algorithms for constructing the low rank separated approximation, evaluating $(L f)(x)$, as well as for evaluating its adjoint, namely, computing $\left(L^{*} f\right)(x)$. Numerical examples in section 4 illustrate the properties of our algorithms. Finally, section 5 discusses some related work and potential alternatives.

2. Analytical estimates. In this section, we return to a description of the problem in continuous variables $x$ and $\xi$ to prove estimates on the separation rank of $e^{2 \pi i \Phi_{\ell}(x, \xi)}$, where $\Phi_{\ell}(x, \xi)$ is the residual phase after linearization about $\hat{\xi}_{\ell}$.

2.1. Background. We begin with a lemma which concerns the separation of the exponential function and whose variations play a central role in modern numerical analysis.

Lemma 1. Consider the domain defined by $x \in[-A, A]$ for some $A>0$, and $y \in[-1,1]$. For all $\epsilon>0$ the $\epsilon$-rank $r_{\epsilon}$ of $e^{i x y}$ on $[-A, A] \times[-1,1]$ obeys the bound $r_{\epsilon} \leq r_{\epsilon}^{*}$, where

$$
r_{\epsilon}^{*}=1+\max \left\{2 e A, \log _{2}\left(2 \epsilon^{-1}\right)\right\} .
$$

Furthermore, if $A \leq \frac{1}{2 e}$, then the stronger bound

$$
r_{\epsilon}^{*}=1+\frac{\log \left(2 \epsilon^{-1}\right)}{\log \frac{1}{e A}}
$$

holds as well. In both cases, the corresponding separated representation is the expansion

$$
\left|e^{i x y}-\sum_{n=0}^{r_{\epsilon}^{*}-1} \frac{i^{n}}{n !} x^{n} y^{n}\right| \leq \epsilon
$$

Proof. The proof is very simple. We start with

$$
\left|e^{i x y}-\sum_{n=0}^{r-1} \frac{(i x y)^{n}}{n !}\right| \leq \sum_{n \geq r} \frac{A^{n}}{n !} \leq \sum_{n \geq r}\left(\frac{e A}{n}\right)^{n} \leq \sum_{n \geq r}\left(\frac{e A}{r}\right)^{n}=\left(\frac{e A}{r}\right)^{r} \frac{1}{1-\frac{e A}{r}} .
$$

If $e A \leq 1 / 2$, then a fortiori $e A / r \leq 1 / 2$, and the condition $r \geq \log \left(2 \epsilon^{-1}\right) / \log \frac{1}{e A}$ allows us to bound

$$
\left(\frac{e A}{r}\right)^{r} \frac{1}{1-\frac{e A}{r}} \leq 2 \cdot(e A)^{r} \leq \epsilon
$$


On the other hand, if $e A \geq 1 / 2$, then we have to impose $e A / r \leq 1 / 2$ by hand, as one of the alternatives of the max in (2.1). Then the extra condition $r \geq \log _{2}\left(2 \epsilon^{-1}\right)$ allows us to bound

$$
\left(\frac{e A}{r}\right)^{r} \frac{1}{1-\frac{e A}{r}} \leq 2 \cdot 2^{-r} \leq \epsilon
$$

Since the $\epsilon$-rank $r_{\epsilon}$ is integer-valued, the estimate on $r$ may need to be rounded up to the next integer - hence the precaution of incrementing the bounds in (2.1) and (2.2) by one.

In the next section we will make use of Lemma 1 to prove that the nonoscillatory factor $e^{2 \pi i \Phi_{\ell}(x, \xi)}$ has a separation rank which is independent of $N$. The other factor in the kernel $a(x, \xi) e^{2 \pi i \Phi_{\ell}(x, \xi)}$, namely, the amplitude $a(x, \xi)$, is in general a simpler object to study. The standard assumption in the literature, and also in applications, is to assume that $a(x, \xi)$ is a smooth symbol of order zero and type $(1,0)$, meaning that for each pair of integers $(\alpha, \beta)$, there is a positive constant $C_{\alpha \beta}$ obeying

$$
\left|\partial_{\xi}^{\alpha} \partial_{x}^{\beta} a(x, \xi)\right| \leq C_{\alpha \beta}\left(1+|\xi|^{2}\right)^{-|\alpha| / 2} .
$$

For simplicity, we will also assume that $a(x, \xi)$ is compactly supported in $x .^{2}$ The nice separation properties of $a$ are simple consequences of its assumed smoothness.

Lemma 2. Assume $a(x, \xi)$ is a symbol of order zero. Then for all $M>0$ there exists $C_{M}>0$ such that for all $\epsilon>0$, the $\epsilon$-rank for the separation of $x$ and $\xi$ in $a(x, \xi)$ obeys

$$
r_{\epsilon} \leq C_{M} \epsilon^{-1 / M}
$$

Proof. Perform a Fourier transform of the $C^{\infty}$, compactly supported function $a(\cdot, \xi)$. It suffices to keep $O\left(\epsilon^{-1 / M}\right)$ Fourier modes to approximate $a(\cdot, \xi)$ to accuracy $\epsilon$ on its compact support. Each Fourier mode is of the form $\hat{a}(\omega, \xi) e^{i \omega x}$ and is hence separated.

It goes without saying that the $\epsilon$-rank of the product $a(x, \xi) e^{2 \pi i \Phi_{\ell}(x, \xi)}$ is bounded by a constant times the product of the individual $\epsilon$-ranks, and we now focus on the real object of interest, the factor $e^{2 \pi i \Phi_{\ell}}$.

2.2. Large $N$ asymptotics. In this section we assume that the phase $\Phi(x, \xi)$ is $C^{3}$ in $\xi$, measurable only in $x$, and we define

$$
C_{k}=2 \pi \sup _{x \in[0,1]^{2}} \sup _{\xi:|\xi|=1}\left|\partial_{\theta}^{k} \Phi(x, \xi)\right| \quad \text { for } 0 \leq k \leq 3,
$$

where $\theta=\arg \xi$. These constants will enter our estimates only through the following combinations:

$$
D_{2}=C_{0}+C_{2} \quad \text { and } \quad D_{3}=C_{1}+C_{3} .
$$

As before, we also require homogeneity of order one in $\xi$. Finally, we let the general angular opening of the cone $W_{\ell}$ be $\frac{2 \alpha}{\sqrt{N}}$ radians for some constant $\alpha$ (the introduction section proposed $\alpha=\pi$ ).

\footnotetext{
${ }^{2}$ This assumption is equivalent to assuming that functions in the range of $L$ are themselves compactly supported in situations of interest, which ought to be the case for accurate numerical computations.
} 
The result below is a more precise version of the theorem we introduced in section 1.

THEOREM 1. For all $0<\epsilon \leq 1$ and $N \geq \frac{\alpha^{6} D_{3}^{2}}{18 \epsilon^{2}}$, the $\epsilon$-separation rank of $e^{2 \pi i \Phi_{\ell}(x, \xi)}$ for $x \in[0,1]^{2}$ and $\xi \in W_{\ell}$ obeys

$$
r_{\epsilon} \leq 1+\max \left\{\frac{e \sqrt{2}}{2} \alpha^{2} D_{2}, \log _{2}\left(4 \epsilon^{-1}\right)\right\} .
$$

Furthermore, if $\alpha$ is admissible in the sense that $\alpha \leq \sqrt{\frac{\sqrt{2}}{e D_{2}}}$, then

$$
r_{\epsilon} \leq 1+\frac{\log \left(4 \epsilon^{-1}\right)}{\log \frac{2 \sqrt{2}}{e \alpha^{2} D_{2}}} .
$$

Proof. Put $r=|\xi|$ and $\theta$ as the angle measured from the vector $\xi_{\ell}$. The phase $\Phi$ can be rewritten as $\Phi(x, \xi)=r \phi(x, \theta)$. Let $\xi_{1}$ be the frequency coordinate along $\xi_{\ell}$ and $\xi_{2}$ orthogonal to $\xi_{1}$, so that we can switch between polar and Cartesian coordinates using

$$
\frac{\partial \Phi}{\partial \xi_{1}}\left(x, \xi_{\ell}\right)=\phi(x, 0) \quad \text { and } \quad \frac{\partial \Phi}{\partial \xi_{2}}\left(x, \xi_{\ell}\right)=\phi^{\prime}(x, 0),
$$

where the derivative of $\phi$ is taken in $\theta$. The residual phase is

$$
\begin{aligned}
\Phi_{\ell}(x, \xi) & =\Phi(x, \xi)-\nabla_{\xi} \Phi\left(x, \xi_{\ell}\right) \cdot \xi \\
& =r\left(\phi(x, \theta)-\cos \theta \phi(x, 0)-\sin \theta \phi^{\prime}(x, 0)\right) .
\end{aligned}
$$

We can now expand $\phi(x, \theta), \cos \theta$, and $\sin \theta$ in a Maclaurin series (around $\theta=0$ ) to obtain

$$
\Phi_{\ell}(x, \xi)=\frac{r \theta^{2}}{2}\left(\phi(x, 0)+\phi^{\prime \prime}(x, 0)\right)+\frac{r \theta^{3}}{6}\left[\phi^{\prime \prime \prime}(x, \bar{\theta})-\cos (\tilde{\theta}) \phi^{\prime}(x, 0)\right]
$$

for some $\tilde{\theta}$ and $\bar{\theta}$ between 0 and $\theta$ (with $\bar{\theta}$ depending on $x$.)

The $x$ and $\xi$ variables are separated in the first term of (2.6), and so we write

$$
f(x) g(\xi) \equiv 2 \pi\left(\phi(x, 0)+\phi^{\prime \prime}(x, 0)\right) \frac{r \theta^{2}}{2} .
$$

The term with square brackets is the remainder, and we write

$$
R(x, \xi)=2 \pi \frac{r \theta^{3}}{6}\left[\phi^{\prime \prime \prime}(x, \bar{\theta})-\cos (\tilde{\theta}) \phi^{\prime}(x, 0)\right] .
$$

Our strategy will be to choose $N$ large enough so that $R(x, \xi)$ becomes negligible; hence only the exponential of the first term needs to be separated.

Recall that in two dimensions the frequency domain is the square $\left[-\frac{N}{2}, \frac{N}{2}-1\right]^{2}$. Since $|\theta| \leq \frac{\alpha}{\sqrt{N}}$ in the wedge $W_{\ell}$, and $r \leq \frac{\sqrt{2}}{2} N$, we have the following bounds for the two terms in (2.6):

$$
|f(x) g(\xi)| \leq \frac{\sqrt{2}}{4} \alpha^{2} D_{2}, \quad|R(x, \xi)| \leq \frac{\sqrt{2}}{12} \frac{\alpha^{3}}{\sqrt{N}} D_{3} .
$$

Copyright $@$ by SIAM. Unauthorized reproduction of this article is prohibited. 
It is instructive to notice that the bound on $|f g|$ is independent of $N$. That is the reason why we chose the angular opening of the cone $W_{\ell}$ proportional to $N^{-1 / 2}$ (parabolic scaling).

The first contribution to the separation remainder is given by

$$
\begin{aligned}
\left|e^{i(f g+R)}-e^{i f g}\right| & =\left|e^{i R}-1\right| \\
& \leq|R| \leq \frac{\sqrt{2}}{12} \frac{\alpha^{3}}{\sqrt{N}} D_{3} .
\end{aligned}
$$

The condition on $N$ ensures precisely that this remainder be dominated by $\epsilon / 2$.

The second contribution to the total error is due to the separation of $e^{i f g}$ itself and needs to be made smaller than $\epsilon / 2$ as well. We invoke Lemma 1 with $f(x) \times \sup |g(\xi)|$ in place of $x, g(\xi) / \sup |g(\xi)|$ in place of $y$, and $\epsilon / 2$ in place of $\epsilon$. With these choices, $A$ becomes $\frac{\sqrt{2}}{4} \alpha^{2} D_{2}$, and we obtain the desired result.

2.3. Small $\epsilon$ asymptotics. Theorem 1 is a special asymptotic result in the case of large $N$ (problem size) - or alternatively small $\alpha$ (cone's angular opening). This regime may not be attained in practice, and so we need another result, without restrictions on $N$ and informative for arbitrarily small $\epsilon$.

To this effect, we need stronger (yet still realistic) smoothness assumptions on the phase $\Phi$ : for each $x$, we require that $\Phi(x, \xi)$ be a real-analytic function of $\xi$. This condition implies the bound

$$
2 \pi \sup _{|\xi|=1}\left|\partial_{\theta}^{k} \Phi(x, \xi)\right| \leq Q k ! R^{-k}
$$

for some constants $Q$ and $R$. For example, $R$ can be taken as any number smaller than the uniform radius of convergence in $\theta$, in which case $Q$ will in general depend on $R$. Let us term such phases, or functions, $(Q, R)$-analytic. As before, we also require homogeneity in $\xi$.

Theorem 2. Assume $\Phi_{\ell}(x, \xi)$ is measurable in $x$ and $(Q, R)$-analytic in $\xi$ for some constants $Q$ and $R$. Assume that $\alpha$ is admissible in the sense that

$$
\alpha<\min \left\{\frac{R \sqrt{N}}{2}, \frac{R}{\sqrt{\sqrt{2} Q}}\right\} .
$$

Then for all $0<\epsilon \leq 1$, the $\epsilon$-separation rank of $e^{2 \pi i \Phi_{\ell}(x, \xi)}$ for $x \in[0,1]^{2}$ and $\xi \in W_{\ell}$ obeys

$$
r_{\varepsilon} \leq C_{p} \epsilon^{-p} \quad \forall p: p>\frac{2}{\log _{2}\left(\frac{R \sqrt{N}}{\alpha}\right)} .
$$

Proof. Throughout the proof, $x \in[0,1]^{2}$ and $\xi \in W_{\ell}$. Using the smoothness assumption on $\Phi_{\ell}$, we can repeat the reasoning of the proof of Theorem 1 and obtain the convergent series

$$
2 \pi \Phi_{\ell}(x, \xi)=\sum_{k=0}^{\infty} f_{k}(x) g_{k}(\xi),
$$

where $f_{k}(x)=2 \pi \phi^{(k)}(x, 0)$ (the differentiations are in $\theta$ ) and

$$
g_{0}(\xi)=r(1-\cos \theta), \quad g_{1}(\xi)=r(\theta-\sin \theta), \quad g_{k}(\xi)=\frac{r \theta^{k}}{k !} .
$$

Copyright $@$ by SIAM. Unauthorized reproduction of this article is prohibited. 
We denote the bound $\left|f_{k}(x) g_{k}(\xi)\right| \leq A_{k}$ with

$$
A_{0}=\frac{\sqrt{2}}{4} Q \alpha^{2}, \quad A_{1}=\frac{\sqrt{2}}{12} Q \frac{\alpha^{3}}{R \sqrt{N}}, \quad A_{k}=\frac{\sqrt{2}}{2} Q N\left(\frac{\alpha}{R \sqrt{N}}\right)^{k} \quad \text { for } k>2 .
$$

Our strategy will be to call upon Lemma 1 for the first few factors $e^{i f_{k} g_{k}}$ in order to obtain a separation rank $r_{k}$ and an error $\epsilon_{k}$ for each of them:

$$
\left|e^{i f_{k} g_{k}}-\sum_{n=0}^{r_{k}-1} \frac{i^{n}}{n !} f_{k}^{n}(x) g_{k}^{n}(\xi)\right| \leq \epsilon_{k} .
$$

We will perform this operation for each $k<K$, with $K$ large enough, to be determined. Once the separation of each factor is available, we can write

$$
e^{i \sum_{k=0}^{K-1} f_{k} g_{k}}=\prod_{k=0}^{K-1} e^{i f_{k} g_{k}}
$$

and obtain the bound on the overall separation rank as the product $\prod_{k=0}^{K-1} r_{k}$.

There are two sources of errors we must contend with:

- Truncation in $k$. The factors $e^{i f_{k} g_{k}}$ for $k \geq K$ will be deemed negligible if their combined contribution results in an overall error smaller than $\epsilon / 2$, meaning

$$
\left|e^{2 \pi i \Phi_{\ell}}-e^{i \sum_{k=0}^{K-1} f_{k} g_{k}}\right| \leq \frac{\epsilon}{2} .
$$

The left-hand side is bounded by $\left|\sum_{K}^{\infty} f_{k} g_{k}\right|$. Using the bound we stated earlier on $A_{k}$ and the admissibility condition on $\alpha$, a bit of algebra shows that $(2.8)$ is satisfied for

$$
K=\left\lceil\frac{\log \left(2 \sqrt{2} Q N \epsilon^{-1}\right)}{\log \left(\frac{R \sqrt{N}}{\alpha}\right)}\right\rceil
$$

(meaning the smallest integer greater than the quotient inside the brackets). This quantity in turn obeys $K \leq \log _{2}\left(16 \epsilon^{-1}\right)$.

- Truncation in $n$. The truncation errors from (2.7) must be made sufficiently small so that their combined contribution also results in an overall error smaller than $\epsilon / 2$, meaning

$$
\left|\prod_{k=0}^{K-1} e^{i f_{k} g_{k}}-\prod_{k=0}^{K-1} \sum_{n=0}^{r_{k}-1} \frac{i^{n}}{n !} f_{k}^{n}(x) g_{k}^{n}(\xi)\right| \leq \frac{\epsilon}{2} .
$$

Easy manipulations ${ }^{3}$ show that (2.10) follows from the bound

\footnotetext{
${ }^{3}$ To justify this step, put $E_{k}(x, \xi)=e^{i f_{k}(x) g_{k}(\xi)}$ and start from the identity

$$
\begin{aligned}
\prod_{k=0}^{K-1}\left(E_{k}+\epsilon_{k}\right)-\prod_{k=0}^{K-1} E_{k} & =\sum_{j} \epsilon_{j} \prod_{k \neq j}\left(E_{k}+\tau_{j k} \epsilon_{k}\right) \\
& =\sum_{j} \epsilon_{j} E_{j}^{-1} \prod_{k=0}^{K-1}\left(E_{k}+\tau_{j k} \epsilon_{k}\right)
\end{aligned}
$$

where $\tau_{j k}=0$ if $j \leq k$, and $\tau_{j k}=1$ if $j>k$. Then make use of the bound $\left(1+\frac{\epsilon}{3 K}\right)^{K}<e^{\epsilon / 3} \leq$ $e^{1 / 3}<3 / 2$.
} 


$$
\epsilon_{k}=\frac{\epsilon}{3 K} .
$$

(Recall that $K$ is comparable to $\log \left(C \epsilon^{-1}\right)$.)

Such a bound holds if, in turn, we take $r_{k}$ large enough. The admissibility condition on $\alpha$ ensures, among others, that we can invoke the strong version of Lemma 1, namely, (2.2), and obtain

$$
r_{k} \leq 1+\frac{\log \left(2 \epsilon_{k}^{-1}\right)}{\log \frac{1}{e A_{k}}} .
$$

It now remains to estimate $\prod_{k=0}^{K-1} r_{k}$, where $r_{k}$ is given by (2.11) and $K$ by (2.9). We treat the first two factors independently. It follows from the bounds on $A_{0}, A_{1}$, and $K$ and the fact that $\alpha$ and $Q$ are constant in $\epsilon$ and $N$ that

$$
\begin{aligned}
r_{0} & \leq 1+\frac{\log \left(6 K \epsilon^{-1}\right)}{\log \frac{4}{e \sqrt{2} Q \alpha^{2}}} \leq C \log \left(6 K \epsilon^{-1}\right) \\
& \leq C \log \left(3 \log \left(16 \epsilon^{-1}\right)\right)+C \log \left(2 \epsilon^{-1}\right) \leq C \log \left(2 \epsilon^{-1}\right)
\end{aligned}
$$

(the constant $C$ changes from expression to expression), and similarly

$$
r_{1} \leq C \log \left(2 \epsilon^{-1}\right) .
$$

The same logarithmic bound holds for $r_{k}$ in the case $k \geq 2$ but will not suffice for our purpose. Instead, we write

$$
\begin{aligned}
r_{k} & \leq 1+\frac{\log \left(6 K \epsilon^{-1}\right)}{\log \left[\frac{\sqrt{2}}{Q N}\left(\frac{R \sqrt{N}}{\alpha}\right)^{k}\right]} \\
& \leq \frac{\log \left(C \epsilon^{-1} \log \left(2 \epsilon^{-1}\right)\right)+k \log \left(\frac{R \sqrt{N}}{\alpha}\right)}{\log (C)+k \log \left(\frac{R \sqrt{N}}{\alpha}\right)} \\
& \equiv \frac{A+k}{B+k} \quad(k \geq 2) .
\end{aligned}
$$

We simplified notations only in the last line. Notice that $A>B$ and that $B+k \geq 1$ when $k \geq 2$. We will assume without loss of generality that $A$ and $B$ are integers. The value of the product $\prod r_{k}$ can increase only if we replace the initial bound $0 \leq k<K$ by the condition that the bound on $r_{k}$ be greater than 2 . So we certainly have

$$
\begin{aligned}
r_{\epsilon} & \leq \prod_{k \geq 2: r_{k} \geq 2} r_{k} \leq \frac{A+2}{B+2} \frac{A+3}{B+3} \cdots \frac{A+A}{B+A} \\
& =\frac{(2 A) ! /(A+1) !}{(B+A) ! /(B+1) !} .
\end{aligned}
$$

We can now make use of the two-sided Stirling bound

$$
\sqrt{2 \pi} n^{n+1 / 2} e^{-n+\frac{12}{n+1}} \leq n ! \leq \sqrt{2 \pi} n^{n+1 / 2} e^{-n+\frac{12}{n}}
$$

Copyright $@$ by SIAM. Unauthorized reproduction of this article is prohibited. 
to obtain

$$
\begin{aligned}
r_{\epsilon} & \leq C \frac{(2 A)^{2 A}(A+1)^{-(A+1)}}{(A+B)^{A+B}(B+1)^{-(B+1)}} \\
& \leq C 2^{2 A} \frac{A^{2 A}}{(A+1)^{(A+1)}(A+B)^{A-1}} \frac{(B+1)^{(B+1)}}{(A+B)^{B+1}} \\
& \leq C 2^{2 A}
\end{aligned}
$$

In turn,

$$
2^{2 A} \leq\left(C \epsilon^{-1} \log \left(2 \epsilon^{-1}\right)\right)^{\frac{2}{\log _{2}\left(\frac{R \sqrt{N}}{\alpha}\right)}}
$$

which concludes the proof.

The lower the fractional exponent of $\epsilon^{-1}$ the faster the convergence of separated expansions. Theorem 2 shows exactly which factors can make this exponent arbitrarily small:

- large grid size $N$, or

- small angular opening constant $\alpha$, or

- large radius of analyticity $R$ of the phase in $\arg \xi$ (uniformly in $x$ ). Observe that the rank bound decreases as $N$ increases.

Theorem 2 assumes that the residual phase function $\Phi_{\ell}(x, \xi)$ is $(Q, R)$-analytic in $\xi$. The variation below follows the same path of reasoning and is useful when $\Phi_{\ell}(x, \xi)$ is only $C^{\infty}$ in $\xi$ for $\xi \neq 0$.

TheOREM 3. Assume $\Phi_{\ell}(x, \xi)$ is $C^{\infty}$ in $\xi$ for $\xi \neq 0$. For any $p>0$, there exist two constants $C_{p}$ and $C_{p}^{\prime}$ such that for any $N$, the $\epsilon$-separation rank with $\varepsilon=C_{p} N^{-p}$ is bounded by $C_{p}^{\prime} \log N$.

Proof. The structure of the proof is similar to that of Theorem 2. One needs only to keep the first $2 p+2$ term of the series

$$
2 \pi \Phi_{\ell}(x, \xi)=\sum_{k=0}^{\infty} f_{k}(x) g_{k}(\xi)
$$

in order to have $\varepsilon=C_{p} N^{-p}$ for some constant $C_{p}$ which depends only on $p$ and $\Phi_{\ell}$. The product $\prod_{k=0}^{2 p+1} r_{k}$ upper bounds the overall separation rank and is less than $C_{p}^{\prime} \log N$ for some constant $C_{p}^{\prime}$ which depends only on $p$.

In many computational problems, the mesh size $N^{-1}$ is linked directly to the desired accuracy $\varepsilon$, usually in the form of a power law, e.g., $\varepsilon=O\left(N^{-p}\right)$ for some constant $p$. Therefore, Theorem 3 is interesting for practical reasons.

3. Algorithm. For notational convenience, we assume in this section that the amplitude is identically equal to one; that is, we focus on the so-called (discretized) Egorov operator

$$
(L f)(x)=\frac{1}{N} \sum_{\xi \in \Omega} e^{2 \pi i \Phi(x, \xi)} \hat{f}(\xi) .
$$

Both in practice (section 4) and in theory (section 2), one can easily take care of general amplitude terms. 
The algorithm for computing (3.1) has two main components:

- Preprocessing step. Given the residual phase $\Phi_{\ell}(x, \xi) \equiv \Phi(x, \xi)-\nabla_{\xi} \Phi\left(x, \hat{\xi}_{\ell}\right) \cdot \xi$, this step constructs, for each wedge $W_{\ell}$, a low rank separated approximation

$$
\left|e^{2 \pi i \Phi_{\ell}(x, \xi)}-\sum_{t=1}^{q} \gamma_{\ell t}^{x}(x) \gamma_{\ell t}^{\xi}(\xi)\right| \leq \varepsilon .
$$

The functions $\left\{\gamma_{\ell t}^{x}(x)\right\}$ and $\left\{\gamma_{\ell t}^{\xi}(\xi)\right\}$, or their compressed versions, are then stored for use in the next step.

- Evaluation step. Given a function $f$, this step computes $(L f)(x)$ approximately by

$$
(L f)(x) \approx \frac{1}{N} \sum_{\ell} \sum_{t} \gamma_{\ell t}^{x}(x) \sum_{\xi} e^{2 \pi i \nabla_{\xi} \Phi\left(x, \hat{\xi}_{e}\right) \cdot \xi}\left[\gamma_{\ell t}^{\xi}(\xi) \chi_{\ell}(\xi) \hat{f}(\xi)\right] .
$$

The preprocessing step is performed only once for a fixed phase function $\Phi(x, \xi)$. The family of functions $\left\{\gamma_{\ell t}^{x}(x)\right\}$ and $\left\{\gamma_{\ell t}^{\xi}(\xi)\right\}$ should of course be used again and again to compute $(L f)(x)$ for different inputs $f$.

In sections 3.1 and 3.2, we propose two different approaches for constructing the families $\left\{\gamma_{\ell t}^{x}(x)\right\}$ and $\left\{\gamma_{\ell t}^{\xi}(\xi)\right\}$. Section 3.4 describes the details of the evaluation step. Finally, section 3.5 outlines the algorithm for rapidly applying the adjoint operator. In this section, we calculate time and storage complexity under the assumption of large grids, i.e., that of Theorem 2. For other kinds of asymptotics, one may need to adjust these estimates with a multiplicative $\log N$ factor, which is typically negligible.

3.1. Preprocessing step: Deterministic approach. We first describe a deterministic approach for constructing the low rank separated expansion, based on a Taylor expansion, exactly as in the proof of Lemma 1 . For each wedge $W_{\ell}$, the strategy consists of the following sequence of steps:

- First, construct a low rank separated approximation of $\Phi_{\ell}(x, \xi)$. This is done by truncating the polar coordinates Taylor expansion to the $(2 p+1)$ st term

$$
\Phi_{\ell}(x, \xi) \approx|\xi| \sum_{k=1}^{2 p+1} c_{\ell k}(x)\left(\theta-\theta_{\ell}\right)^{k} .
$$

Here $p$ is a constant that determines the level of accuracy.

- Second, for each $k$ construct a separated expansion of $e^{2 \pi i c_{\ell k}(x)|\xi|\left(\theta-\theta_{\ell}\right)^{k}}$. This is done by truncating the Taylor expansion to the first $d_{\ell k}$ terms

$$
e^{2 \pi i c_{\ell k}(x)|\xi|\left(\theta-\theta_{\ell}\right)^{k}} \approx \sum_{m=0}^{d_{\ell k}-1} \beta_{\ell k m}^{x}(x) \beta_{\ell k m}^{\xi}(\xi) .
$$

The value of each $d_{\ell k}$ is also chosen to obtain a good accuracy.

- Third, combine the separated expansions for $k=1, \ldots, 2 p+1$ into one separated representation for $e^{2 \pi i \Phi_{\ell}(x, \xi)}$. Simply expanding the product of the expansions obtained in the previous step would be sufficient for proving a theorem like those presented in section 2, but in practice, though, the number of terms in the expansion is too large and far from optimal. We thus combine the product of separated expansions two-by-two with the compression procedure to be described next and repeat the process until there is only one separated expansion left. The final expansion provides us with the required functions $\left\{\gamma_{\ell t}^{x}(x)\right\}$ and $\left\{\gamma_{\ell t}^{\xi}(\xi)\right\}$. 
The compression procedure used to combine the product of two separated expansions is quite standard. Suppose we have only two expansions (the subscript $\ell$ is implicit) and write their product as

$$
\begin{aligned}
\left(\sum_{m_{1}=0}^{d_{1}-1} \beta_{1 m_{1}}^{x}(x) \beta_{1 m_{1}}^{\xi}(\xi)\right) & \left(\sum_{m_{2}=0}^{d_{2}-1} \beta_{2 m_{2}}^{x}(x) \beta_{2 m_{2}}^{\xi}(\xi)\right) \\
= & \sum_{m_{1}, m_{2}}\left(\beta_{1 m_{1}}^{x}(x) \beta_{2 m_{2}}^{x}(x)\right)\left(\beta_{1 m_{1}}^{\xi}(\xi) \beta_{2 m_{2}}^{\xi}(\xi)\right):=\sum_{m} c_{m}^{x}(x) c_{m}^{\xi}(\xi) .
\end{aligned}
$$

We adopt the matrix notation and introduce

$$
(A)_{x, m}=c_{m}^{x}(x), \quad\left(B^{*}\right)_{m, \xi}=c_{m}^{\xi}(\xi) .
$$

The problem is to find two matrices $\tilde{A}$ and $\tilde{B}$ which have far fewer columns than $A$ and $B$ yet obey $\tilde{A} \tilde{B}^{*} \approx A B^{*}$. This may be achieved by means of the QR factorization and the SVD:

1. Construct QR factorizations $A=Q_{A} R_{A}$ and $B=Q_{B} R_{B}$.

2. Compute the SVD of $R_{A} R_{B}^{*}$ and truncate the singular values below a threshold $\varepsilon$ together with their associated left and right singular vectors, i.e., $R_{A} R_{B}^{*} \approx$ $U_{M} S_{M} V_{M}^{*}$, where $S_{M}$ is a truncated diagonal matrix of singular values.

3. Set $\tilde{A}=Q_{A} U_{M} S_{M}$ and $\tilde{B}=Q_{B} V_{M}$.

Suppose $A$ is $m \times q$ and $B$ is $n \times q$ with both $m$ and $n$ much larger than $q$. The computational complexity of the compression procedure is $O\left((m+n) q^{2}\right)$. In our setup, $m=|X|=N^{2}, n=\left|W_{\ell}\right|=O\left(N^{1.5}\right)$, and $q$, the rank bound, is uniformly bounded in $N$ (Theorem 2 shows that $q$ is bounded by a small fractional power of $\varepsilon$, independently of $N$ ). Therefore, the complexity of a single compression procedure is $O\left(N^{2}\right)$. Since this needs to be carried out $2 p-1$ times for each of the $\sqrt{N}$ wedges, the overall complexity of the deterministic preprocessing is $O\left(\sqrt{N} \times N^{2}\right)=O\left(N^{2.5}\right)$, where the constant is directly related to the rank bounds of section 2 .

Next, let us consider the storage requirement. For each wedge, the size of the final separated expansion is $O\left(N^{2}\right)$. Since there are $\sqrt{N}$ wedges, the total storage requirement is $O\left(N^{2.5}\right)$, which can be costly when $N$ is large. For example, in a typical problem with $N=1024$ and $q=20$, the total storage would be about 10 GB assuming double precision is used. Our second approach to solving the preprocessing step addresses this issue and requires dramatically less storage space.

3.2. Preprocessing step: Randomized approach. This section describes a randomized approach for computing the functions $\left\{\gamma_{\ell t}^{x}(x)\right\}$ and $\left\{\gamma_{\ell t}^{\xi}(\xi)\right\}$ for a fixed $\ell$. The method is based on the work presented in Kapur and Long [30]. We use matrix notation and set $A$ to be the matrix defined by

$$
A_{x, \xi}:=e^{2 \pi i \Phi_{\ell}(x, \xi)}, \quad x \in X, \xi \in W_{\ell} .
$$

The matrix $A$ is $m$ by $n$ with $m=N^{2}$ and $n=O\left(N^{1.5}\right)$. Assume the prescribed error $\varepsilon$ is fixed. Theorem 2 tells us that there exists a low rank factorization of $A$ with rank $r_{\varepsilon}=O(1)$ (again, by this we mean that $r_{\varepsilon}$ is bounded by a constant independent of $N$, although not independent of $\epsilon$ ). Using this knowledge, the following randomized method finds an approximate factorization

$$
A \approx U T,
$$

where $U$ is of size $m \times q, T$ is $q \times n$, and $q=O(1)$ in $N$. 
- Select a set $C$ of $r$ columns taken from $A$ uniformly at random, and define $A_{[C]}$ to be the submatrix formed by these columns. In practice, a safe choice is to take $r$ about three times larger than the (unknown) $r_{\varepsilon}$.

- Compute the SVD $A_{[C]} \approx U S V^{*}$, where the diagonal of $S$ contains only the singular values greater than the threshold $\varepsilon$. Since $A$ has a separation rank $r_{\varepsilon}=O(1)$, we expect $U$ to be of size $m \times q$, where $q$ is about $r_{\epsilon}$.

- Select a set $R$ of $r$ rows taken from $A$ uniformly at random, and define $A_{[R]}$ to be the submatrix formed by these rows. Similarly, let $U_{[R]}$ be the submatrix of $U$ containing the same rows.

- Set $T=U_{[R]}^{+} A_{[R]}$, where $U_{[R]}^{+}$is the pseudoinverse of $U_{[R]}$.

- The matrices $U$ and $T$ provide an approximate factorization, i.e., $A \approx U T$. We identify the columns of $U$ with the family $\left\{\gamma_{\ell t}^{x}(x)\right\}$ and the rows of $T$ with $\left\{\gamma_{\ell t}^{\xi}(\xi)\right\}$.

This randomized approach works well in practice, although we are not able to offer a rigorous proof of its accuracy and expect one to be nontrivial. We merely argue that the validity of this methodology hinges on the following observations:

- First, the columns of $A$ are highly correlated. Following the arguments in section 2 , it is not difficult to show that a pair of columns with nearby values of the frequency index $\xi \in W_{\ell}$ has a large inner product. Therefore, as we sample uniformly at random, we get good coverage of the set $W_{\ell}$ (leaving no large hole), and as a result, the sampled columns nearly span the space generated by the columns of $A$. Note that one could also use a deterministic regular sampling strategy; for instance, we could take a Cartesian subgrid as a subset of $W_{\ell}$. We observed that, in practice, the probabilistic approach provides slightly better approximations.

- As the SVD routine is numerically stable, it allows us to extract an orthobasis of the column space of $A_{[C]}$ in a robust way.

- By construction, the columns of $U$ are orthonormal. Results from random projection and the geometry of high-dimensional spaces imply that, as long as $U$ does not correlate with the canonical orthobasis, the columns of $U_{[R]}$ are almost orthogonal as well. This allows us to recover the matrix $T$ in a stable and robust fashion.

The computational complexity of this randomized approach is quite low. The SVD step has a complexity of $O\left(m r^{2}\right)=O\left(N^{2}\right)$, while the matrix product $T=$ $U_{[R]}^{+} A_{[R]}$ takes $O(n r q)=O\left(N^{1.5}\right)$ operations. Therefore, for each $\ell$, the complexity of the randomized approach is $O\left(N^{2}\right)$. Since the same procedure needs to be carried out for all the $\sqrt{N}$ wedges, the overall complexity is $O\left(N^{2.5}\right)$.

Often we do not know the exact value of $r_{\varepsilon}$. Instead of setting $r$ conservatively to be an unnecessarily large number, this difficulty is addressed as follows: we begin with a small $r$, and check whether $q$ is significantly smaller than $r$. If this is the case, we accept the factorization. Otherwise, we double $r$ and restart the process. Geometrical increase guarantees that the work wasted (due to unsuccessful attempts) is bounded by the work of the final successful attempt. In practice, we accept the result when $q \leq r / 3$, and this criterion seems to work well in our numerical experiments. A more conservative test certainly improves the reliability of the factorization but increases the running time.

We finally examine the storage requirement. A naive approach is to store the matrices $U$ and $T$ for each wedge $W_{\ell}$. As $T$ is much smaller than $U$ in size, the storage requirement for each wedge is roughly the size of $U$, which is $N^{2} q=O\left(N^{2}\right)$. 
TABLE 1

Comparison of the deterministic and randomized approaches.

\begin{tabular}{|c|cc|}
\hline & Time & Storage \\
\hline Randomized & $O\left(N^{2.5}\right)$ (small constant) & $O(\sqrt{N})$ \\
Deterministic & $O\left(N^{2.5}\right)$ (large constant) & $O\left(N^{2.5}\right)$ \\
\hline
\end{tabular}

Multiplying this by the number of wedges gives a total storage requirement of $O\left(N^{2.5}\right)$, which can be quite costly for large $N$, as already mentioned in the last section. We propose to store the matrices $V S^{-1}$ and $U_{[R]}^{+}$instead. Both matrices require only storage of size $O(r q)=O(1)$. Whenever we need $U$ and $T$, we form the products $U=A_{[C]} V S^{-1}$ and $T=U_{[R]}^{+} A_{[R]}$. Note that the elements of the matrices $A_{[C]}$ and $A_{[R]}$ are given explicitly by the formula (3.2), and there is of course no need to store them at all. Putting it differently, we rewrite the computed factorization as

$$
A \approx A_{[C]} V S^{-1} U_{[R]}^{+} A_{[R]}
$$

and store only the matrices $V S^{-1}$ and $U_{[R]}^{+}$.

We would like to point out that such a scheme is not likely to work for the deterministic approach. The main reason is that the deterministic approach involves multiple compression procedures which make use of QR factorizations and SVD decompositions. These numerical linear algebra routines are quite complicated, and therefore, it would be difficult to relate the resulting low rank factorization with the elements of the matrix $A$, which have the simple form (3.2).

3.3. Comparison. Table 1 compares the deterministic and the randomized approaches in view of the computational complexity and storage requirement. The deterministic approach has the advantage of guaranteeing an accurate low rank separation. However, the constant in the time complexity can be quite large, as for each wedge, it requires $2 p$ compression procedures to combine multiple separated expansions into a single one. Moreover, since the compression step uses QR factorizations and SVDs, we are forced to store the final expansion, which can be quite costly for large $N$. In practice, the randomized approach constructs a near-optimal low rank expansion with very high probability, requires very low storage space, and enjoys a significantly lower constant in time complexity since it does not utilize repeated QR factorizations or SVDs.

3.4. Evaluation step. Once the families $\left\{\gamma_{\ell t}^{x}(x)\right\}$ and $\left\{\gamma_{\ell t}^{\xi}(\xi)\right\}$ are available, we use the approximation

$$
(L f)(x) \approx \frac{1}{N} \sum_{\ell} \sum_{t} \gamma_{\ell t}^{x}(x) \sum_{\xi} e^{2 \pi i \nabla_{\xi} \Phi\left(x, \hat{\xi}_{\ell}\right) \cdot \xi}\left[\gamma_{\ell t}^{\xi}(\xi) \chi_{\ell}(\xi) \hat{f}(\xi)\right]
$$

to evaluate $L f(x)$. The algorithm simply carries out the evaluation step by step:

1. Compute $\hat{f}$, the Fourier transform of $f$.

2. For each $\ell$ and $t$, form $\hat{f}_{\ell t}(\xi):=\gamma_{\ell t}^{\xi}(\xi) \chi_{\ell}(\xi) \hat{f}(\xi)$.

3. For each $\ell$ and $t$, compute $g_{\ell t}(x):=\sum_{\xi} e^{2 \pi i \nabla_{\xi} \Phi\left(x, \hat{\xi}_{\ell}\right) \cdot \xi} \hat{f}_{\ell t}(\xi)$.

4. Compute $(L f)(x) \approx \frac{1}{N} \sum_{\ell} \sum_{t} \gamma_{\ell t}^{x}(x) g_{\ell t}(x)$.

The only step that requires attention is the third: it asks to evaluate the Fourier series $\sum_{\xi} e^{2 \pi i \nabla_{\xi} \Phi\left(x, \hat{\xi}_{\ell}\right) \cdot \xi} \hat{f}_{\ell t}(\xi)$ at the $N^{2}$ points $\left\{\nabla_{\xi} \Phi\left(x, \hat{\xi}_{\ell}\right): x \in X\right\}$. Even though $X$ is a Cartesian grid, the warped grid $\left\{\nabla_{\xi} \Phi\left(x, \hat{\xi}_{\ell}\right): x \in X\right\}$ is no longer so. In 
fact, the formula for $g_{\ell t}$ is a nonuniform Fourier transform of the second kind, a subject of considerable attention $[2,5,25,34,35]$ since the seminal paper of Dutt and Rokhlin [21]. We adopt the approach introduced in the latter paper and, following their notation, set

- $m=4, q=8$, and $b=0.425$ for 6 digits of accuracy,

- $m=4, q=16$, and $b=0.785$ for 11 digits of accuracy.

We specify these parameter values because they impact the numerical accuracies we will report in the next section, and because it will help anyone interested in reproducing our results.

The algorithm in [21] generally assumes that the Fourier coefficients are supported on the full grid $\Omega$ which is symmetric with respect to the origin. For each $\ell$, the support of $\hat{f}_{\ell t}(\xi)$ is $W_{\ell}$, which is to say that most of the values of the input on the grid $\Omega$ are zero. To speed up the nonuniform FFT, each wedge $W_{\ell}$, which is close to either one of the diagonals, is sheared by 45 degrees so that it becomes approximately horizontal or vertical. Notice that 45 degree shearing of $\hat{f}_{\ell t}(\xi)$ is a simple relabeling of the array. In addition, all wedges are then translated so that their support fits in a rectangle of smaller volume centered around the origin. As the nonuniform FFT [21] asks to compute the FFT of the input data (and then finds a way of interpolating the result on an unstructured grid), we gain efficiency since the input array is now of smaller size. Mathematically, the shearing operation takes the form

$$
\xi^{\prime}=M \xi-\xi_{c},
$$

where $M$ is either the identity or a 45 -degree shear matrix and $\xi_{c}$ is a translation parameter. Thus, we organize the computations as in

$$
\begin{aligned}
\sum_{\xi} e^{2 \pi i \nabla_{\xi} \Phi\left(x, \hat{\xi}_{\ell}\right) \cdot \xi} \hat{f}_{\ell t}(\xi) & =\sum_{\xi^{\prime}} e^{2 \pi i \nabla_{\xi} \Phi\left(x, \hat{\xi}_{\ell}\right) \cdot M^{-1}\left(\xi^{\prime}+\xi_{c}\right)} \hat{f}_{\ell t}\left(M^{-1}\left(\xi^{\prime}+\xi_{c}\right)\right) \\
& =e^{2 \pi i \nabla_{\xi} \Phi\left(x, \hat{\xi}_{\ell}\right) \cdot M^{-1} \xi_{c}} \sum_{\xi^{\prime}} e^{2 \pi i \nabla_{\xi} \Phi\left(x, \hat{\xi}_{\ell}\right) \cdot M^{-1} \xi^{\prime}} \hat{f}_{\ell t}\left(M^{-1}\left(\xi^{\prime}+\xi_{c}\right)\right),
\end{aligned}
$$

where the final summation is a nonuniform Fourier transform at the set of points $\left(M^{*}\right)^{-1} \nabla_{\xi} \Phi\left(x, \hat{\xi}_{\ell}\right)$. In condensed form, the oscillatory modes of the function we wish to evaluate are centered around a center frequency; we factor out this frequency, interpolate the residual, and add the factor back in; for the same accuracy, interpolating the smoother residual requires a smaller computational effort.

A two-dimensional nonuniform FFT takes $O\left(N^{2} \log N\right)$ operations. This operation needs to be repeated $q=O(1)$ times for each one of the $\sqrt{N}$ wedges. Therefore, the overall complexity is $O\left(N^{2.5} \log N\right)$.

3.5. Evaluating the adjoint operator. We conclude this section by presenting how to rapidly apply the adjoint FIO. Begin by expanding the Fourier transform in (1.1) and write

$$
(L f)(x)=\int\left(\int e^{2 \pi i(\Phi(x, \xi)-y \cdot \xi)} \mathrm{d} \xi\right) f(y) \mathrm{d} y
$$

for $x, y, \xi \in \mathbb{R}^{2}$. The adjoint operator is then given by

$$
\begin{aligned}
\left(L^{*} f\right)(x) & =\int\left(\int e^{-2 \pi i(\Phi(y, \xi)-x \cdot \xi)} \mathrm{d} \xi\right) f(y) \mathrm{d} y \\
& =\int\left(\int e^{-2 \pi i \Phi(y, \xi)} f(y) \mathrm{d} y\right) e^{2 \pi i x \cdot \xi} \mathrm{d} \xi
\end{aligned}
$$

Copyright $@$ by SIAM. Unauthorized reproduction of this article is prohibited. 
or equivalently as

$$
\widehat{\left(L^{*} f\right)}(\xi)=\int e^{-2 \pi i \Phi(y, \xi)} f(y) \mathrm{d} y
$$

in the Fourier domain. Similarly, one readily checks that the adjoint of the discretetime FIO is given by the formula

$$
\widehat{\left(L^{*} f\right)}(\xi)=\frac{1}{N} \sum_{y} e^{-2 \pi i \Phi(y, \xi)} f(y)
$$

where $\xi \in \Omega$ and $y \in X$.

Now follow the same set of ideas as in section 3.4, and decompose $L^{*}$ as

$$
\begin{aligned}
\widehat{\left(L^{*} f\right)}(\xi) & =\frac{1}{N} \sum_{\ell} \chi_{\ell}(\xi) \sum_{y} e^{-2 \pi i \Phi(y, \xi)} f(y) \\
& =\frac{1}{N} \sum_{\ell} \chi_{\ell}(\xi) \sum_{y} e^{-2 \pi i \Phi_{\xi}\left(y, \hat{\xi}_{\ell}\right) \cdot \xi} e^{-2 \pi i \Phi_{\ell}(y, \xi)} f(y) \\
& =\frac{1}{N} \sum_{\ell} \chi_{\ell}(\xi) \sum_{y} e^{-2 \pi i \Phi_{\xi}\left(y, \hat{\xi}_{\ell}\right) \cdot \xi} \sum_{t} \overline{\gamma_{\ell t}^{x}(y) \gamma_{\ell t}^{\xi}(\xi)} f(y) \\
& =\frac{1}{N} \sum_{\ell} \sum_{t} \chi_{\ell}(\xi) \overline{\gamma_{\ell t}^{\xi}(\xi)} \sum_{y} e^{-2 \pi i \Phi_{\xi}\left(y, \hat{\xi}_{\ell}\right) \cdot \xi}\left(\overline{\gamma_{\ell t}^{x}(y)} f(y)\right) .
\end{aligned}
$$

The right-hand side of the last equation provides the key steps of the algorithm.

1. For each $\ell$ and $t \leq q$, compute $f_{\ell t}(y):=\overline{\gamma_{\ell t}^{x}(y)} f(y)$.

2. For each $\ell$ and $t \leq q$, compute $g_{\ell t}(\xi):=\sum_{y} e^{-2 \pi i \Phi_{\xi}\left(y, \hat{\xi}_{\ell}\right) \cdot \xi} f_{\ell t}(y)$ using the nonuniform FFT of the first kind; see [21, 25] for details.

3. Compute $\widehat{\left(L^{*} f\right)}(\xi) \approx \frac{1}{N} \sum_{\ell} \sum_{t} \chi_{\ell}(\xi) \overline{\gamma_{\ell t}^{\xi}(\xi)} g_{\ell t}(\xi)$.

4. Finally, take an inverse two-dimensional FFT to get $\left(L^{*} f\right)(x)$.

Clearly, all the results and discussions concerning the matrix vector product $L f$ apply here as well.

4. Numerical results. This section presents several numerical examples to demonstrate the effectiveness of the algorithms introduced in section 3. Our implementation is in MATLAB and all the computational results we are about to report were obtained on a desktop computer with a $2.6 \mathrm{GHz} \mathrm{CPU}$ and $3 \mathrm{~GB}$ of memory. We have implemented both the deterministic and randomized approaches for the preprocessing step. We choose to report the timing and accuracy results of the randomized approach only since it requires less time and storage, as shown in section 3.2.

We first study the error of the separated approximation generated by the randomized preprocessing step. For $x=\left(x_{1}, x_{2}\right)$ and $\xi=\left(\xi_{1}, \xi_{2}\right)$, set the phase function to be

$$
\Phi_{ \pm}(x, \xi)=x \cdot \xi \pm \sqrt{r_{1}^{2}(x) \xi_{1}^{2}+r_{2}^{2}(x) \xi_{2}^{2}} .
$$

We show in the appendix that the transformation, which for each $x$ integrates $f$ along an ellipse centered at $x$ and with axes of length $r_{1}(x)$ and $r_{2}(x)$, can be cast as a sum $L_{+}+L_{-}$of two FIOs given by

$$
\left(L_{ \pm} f\right)(x)=\int a_{ \pm}(x, \xi) e^{2 \pi i \Phi_{ \pm}(x, \xi)} \hat{f}(\xi) \mathrm{d} \xi
$$

Copyright $@$ by SIAM. Unauthorized reproduction of this article is prohibited. 
TABLE 2 proach.

Relative errors of the low rank separated representation constructed using the randomized ap-

\begin{tabular}{|c|cccc|}
\hline & $\varepsilon=1 \mathrm{e}-3$ & $\varepsilon=1 \mathrm{e}-4$ & $\varepsilon=1 \mathrm{e}-5$ & $\varepsilon=1 \mathrm{e}-6$ \\
\hline$N=64$ & $3.57 \mathrm{e}-04$ & $4.93 \mathrm{e}-05$ & $3.21 \mathrm{e}-06$ & $5.17 \mathrm{e}-07$ \\
$N=128$ & $3.11 \mathrm{e}-04$ & $2.28 \mathrm{e}-05$ & $4.19 \mathrm{e}-06$ & $5.81 \mathrm{e}-07$ \\
$N=256$ & $2.85 \mathrm{e}-04$ & $2.83 \mathrm{e}-05$ & $2.94 \mathrm{e}-06$ & $4.13 \mathrm{e}-07$ \\
$N=512$ & $1.66 \mathrm{e}-04$ & $2.82 \mathrm{e}-05$ & $4.38 \mathrm{e}-06$ & $6.80 \mathrm{e}-07$ \\
\hline
\end{tabular}

and with phases obeying (4.1).

In our numerical example, we consider the phase $\Phi_{+}$and choose

$$
\begin{aligned}
& r_{1}(x)=\frac{1}{9}\left(2+\sin \left(4 \pi x_{1}\right)\right)\left(2+\sin \left(4 \pi x_{2}\right)\right), \\
& r_{2}(x)=\frac{1}{9}\left(2+\cos \left(4 \pi x_{1}\right)\right)\left(2+\cos \left(4 \pi x_{2}\right)\right) .
\end{aligned}
$$

In each wedge $W_{\ell}$, the phase is then linearized and a low rank separated approximation $U T$ of the matrix

$$
A=\left(e^{2 \pi i \Phi_{\ell}(x, \xi)}\right)_{x \in X, \xi \in W_{\ell}}
$$

is computed. To estimate the approximation error, we randomly select two sets $\Gamma$ and $\Delta$ of $s$ rows and $s$ columns. Put $A_{\Gamma \Delta}$ to be the $s$ by $s$ submatrix of $A$ with these rows and columns. The separated rank approximation to $A_{\Gamma \Delta}$ is then obtained by multiplying $U_{\Gamma}$ and $T_{\Delta}$, where $U_{\Gamma}$ is the submatrix of $U$ with rows in $\Gamma$ and $T_{\Delta}$ is that of $T$ with columns in $\Delta$. The error is then estimated via

$$
\frac{\left\|A_{\Gamma \Delta}-U_{\Gamma} T_{\Delta}\right\|_{F}}{\left\|A_{\Gamma \Delta}\right\|_{F}},
$$

where $\|\cdot\|_{F}$ stands for the Frobenius norm. In our numerical test, we set $s$ to be 200, and Table 2 displays approximation errors for different combinations of problem size $N$ and accuracy $\varepsilon$. The results show that the randomized approach works quite well and that the estimated error is controlled well below the threshold $\varepsilon$.

Next, consider the relationship between the separation rank and the threshold $\varepsilon$. Theorem 3 shows that $\varepsilon$ scales like $N^{-p}$ for a fixed constant $p$ provided that the separation rank grows gently like $p \log N$. In this experiment, we use the same phase function $\Phi(x, \xi)$ in (4.1) and show the separation rank for different values of $N$ and $p$ in Table 3 . These results suggest that the separation rank is roughly proportional to both $p$ and the logarithm of $N$, which is compatible with the theoretical estimate. Moreover, when $N$ is fixed, the rank seems to grow linearly with respect to $p$, which possibly implies that the constant $C(p)$ in Theorem 3 in fact grows linearly with respect to $p$.

We would like to point out that the number of wedges affects the complexity of our algorithm in two ways. On the one hand, it is obvious from section 3.4 that the complexity grows with the number of wedges in a linear fashion. On the other hand, if we lower the number of wedges, the angular aperture of each wedge has to increase. This implies an increase in the separation rank, which results in a growing computational complexity. In our experiments (Tables 2 and 3), we set the number of wedges to be $\lceil\sqrt{2 N}\rceil$, which, in practice, balances these two competing factors. 
TABLE 3

Ranks of the separated representation generated by the randomized approach for different values of $N$ and $p$. The prescribed error is equal to $N^{-p}$.

\begin{tabular}{|c|rcccc|}
\hline & $p=1$ & $p=1.5$ & $p=2$ & $p=2.5$ & $p=3$ \\
\hline$N=64$ & 7 & 10 & 14 & 18 & 22 \\
$N=128$ & 9 & 12 & 17 & 21 & 24 \\
$N=256$ & 9 & 12 & 17 & 21 & 25 \\
$N=512$ & 10 & 15 & 19 & 24 & 27 \\
\hline
\end{tabular}

TABLE 4

Numerical evaluation of $L f(x)$ with $f$ a two-dimensional white-noise array. The second and third columns give the number of seconds spent in the preprocessing and evaluation steps, respectively. The fourth column shows the speedup factor over the naive algorithm for computing $(L f)(x)$ using the direct summation (4.3). The fifth column is the estimated relative error, and the last gives the amount of memory used in terms of megabytes.

\begin{tabular}{|c|ccccc|}
\hline$(N, \varepsilon)$ & Preprocessing & Evaluation & Speedup & Error & Storage \\
\hline$(64,2.44 \mathrm{e}-03)$ & $2.06 \mathrm{e}+00 s$ & $3.89 \mathrm{e}+00 s$ & $2.05 \mathrm{e}+00$ & $2.08 \mathrm{e}-03$ & $0.76 M$ \\
$(128,6.10 \mathrm{e}-04)$ & $1.09 \mathrm{e}+01 s$ & $2.45 \mathrm{e}+01 s$ & $6.58 \mathrm{e}+00$ & $8.02 \mathrm{e}-04$ & $1.26 M$ \\
$(256,1.53 \mathrm{e}-04)$ & $8.10 \mathrm{e}+01 s$ & $1.65 \mathrm{e}+02 s$ & $1.67 \mathrm{e}+01$ & $1.00 \mathrm{e}-04$ & $2.01 M$ \\
$(512,3.81 \mathrm{e}-05)$ & $4.67 \mathrm{e}+02 s$ & $9.88 \mathrm{e}+02 s$ & $4.46 \mathrm{e}+01$ & $4.22 \mathrm{e}-05$ & $3.06 M$ \\
\hline
\end{tabular}

We now turn to the numerical evaluation of $(L f)(x)$,

$$
(L f)(x)=\frac{1}{N} \sum_{\xi} e^{2 \pi i \Phi(x, \xi)} \hat{f}(\xi),
$$

where the phase function $\Phi$ is the same as in (4.1). In this example, $f$ is an array of independently and identically mean-zero normal random variables (Gaussian white noise), which in some ways is the most challenging input. The threshold $\varepsilon$ is set to be $10 N^{-2}$ (i.e., $p=2$ ). To estimate the error, we first pick $s$ points $\left\{x_{i}: i=1, \ldots, s\right\}$ from $X$ and put $\left\{\widetilde{(L f)}\left(x_{i}\right)\right\}$ for the output of our algorithm (section 3.4). We then compare the values of $\widetilde{(L f)}\left(x_{i}\right)$ at these points with those of $\left\{(L f)\left(x_{i}\right)\right\}$ obtained by evaluating (4.3) directly. Finally, we estimate the relative error with

$$
\sqrt{\frac{\sum_{i}\left|(L f)\left(x_{i}\right)-\widetilde{(L f)}\left(x_{i}\right)\right|^{2}}{\sum_{i}\left|(L f)\left(x_{i}\right)\right|^{2}}} .
$$

Here, we choose $s=100$, and Table 4 summarizes our findings for various values of $N$. The results show that our algorithm performs well. The error is controlled well below threshold and the speedup over the naive algorithm is significant for large values of $N$.

We have considered only the evaluation of FIOs in "Egorov" form thus far (constant amplitude), but the algorithm described in section 3 can be easily extended to operate with general amplitudes provided that the term $a(x, \xi)$ also admits a low rank separated representation in the variables $x$ and $\xi$.

To study the performance of our algorithm in the more general setup of variable amplitudes, we continue with the example where $f$ is integrated along ellipses (4.2) (recall the phase (4.1)). The appendix shows that a possible choice for the amplitudes $a_{ \pm}(x, \xi)$ and phases $\Phi_{ \pm}(x, \xi)$ is

$$
\begin{aligned}
a_{ \pm}(x, \xi) & =\frac{1}{4 \pi}\left(J_{0}(2 \pi \rho(x, \xi)) \pm i Y_{0}(2 \pi \rho(x, \xi))\right) e^{\mp 2 \pi i \rho(x, \xi)} \\
\Phi_{ \pm}(x, \xi) & =x \cdot \xi \pm \rho(x, \xi)
\end{aligned}
$$

Copyright $@$ by SIAM. Unauthorized reproduction of this article is prohibited. 
TABLE 5

Numerical evaluation of $L f(x)$ with $f$ a two-dimensional white-noise array.

\begin{tabular}{|c|ccccc|}
\hline$(N, \varepsilon)$ & Preprocessing & Evaluation & Speedup & Error & Storage \\
\hline$(64,2.44 \mathrm{e}-03)$ & $2.18 \mathrm{e}+01 s$ & $3.67 \mathrm{e}+01 s$ & $4.54 \mathrm{e}+00$ & $7.30 \mathrm{e}-04$ & $0.37 M$ \\
$(128,6.10 \mathrm{e}-04)$ & $1.09 \mathrm{e}+02 s$ & $1.65 \mathrm{e}+02 s$ & $1.49 \mathrm{e}+01$ & $4.00 \mathrm{e}-04$ & $0.59 M$ \\
$(256,1.53 \mathrm{e}-04)$ & $6.62 \mathrm{e}+02 s$ & $8.46 \mathrm{e}+02 s$ & $4.49 \mathrm{e}+01$ & $1.39 \mathrm{e}-04$ & $0.89 M$ \\
$(512,3.81 \mathrm{e}-05)$ & $3.42 \mathrm{e}+03 s$ & $4.43 \mathrm{e}+03 s$ & $1.62 \mathrm{e}+02$ & $3.69 \mathrm{e}-05$ & $1.38 M$ \\
\hline
\end{tabular}

with

$$
\rho(x, \xi)=\sqrt{r_{1}^{2}(x) \xi_{1}^{2}+r_{2}^{2}(x) \xi_{2}^{2}}
$$

Here, $J_{0}$ and $Y_{0}$ are Bessel functions of the first and second kinds, respectively; see the appendix for details.

For the axes lengths, set

$$
r_{1}(x)=r_{2}(x) \equiv r(x)=\frac{1}{16}\left(3+\sin \left(4 \pi x_{1}\right)\right)\left(3+\sin \left(4 \pi x_{2}\right)\right)
$$

(which means that our ellipses are circles). We compute $\left(L_{+} f\right)(x)$ for different values of $N$ and $\varepsilon$ and provide the results in Table 5 . The computational analysis shows that our algorithm performs equally well in the variable amplitude case. For $N=512$, the speedup factor over the naive evaluation is about 162. It is clear from section 3.4 that the dominant part of the evaluation step is the $O(q \sqrt{N})$ calls to the nonuniform FFT. An improved implementation of the nonuniform FFT would certainly result in greater speedup factors.

An extremely important property of Fourier integral operators is that, under the nondegeneracy condition

$$
\operatorname{det}\left(\frac{\partial^{2} \Phi}{\partial x_{i} \partial \xi_{j}}\right) \neq 0
$$

the composition of a FIO with its adjoint preserves the singularities of the input function. Mathematically speaking, if $W F(f)$ is the wave front set of $f[20,39]$, then

$$
W F\left(L^{*} L f\right)=W F(f) .
$$

This property serves as the foundation for most of the current imaging techniques in reflection seismology [39]. In the final example of this section, we verify this phenomenon numerically. We choose the phase function to be

$$
\Phi(x, \xi)=x \cdot \xi+r(x)|\xi|
$$

where $r(x)$ is given by (4.6), and compute $\left(L^{*} L f\right)$ using the algorithm discussed in sections 3.4 and 3.5. Figure 2 displays results for three input functions with different kinds of singularities. Looking at the picture, we see that the "singularities" of $L f$ are of course different than those of $f$, but we also see that the "singularities" of $L^{*} L f$ coincide with those of $f$. 

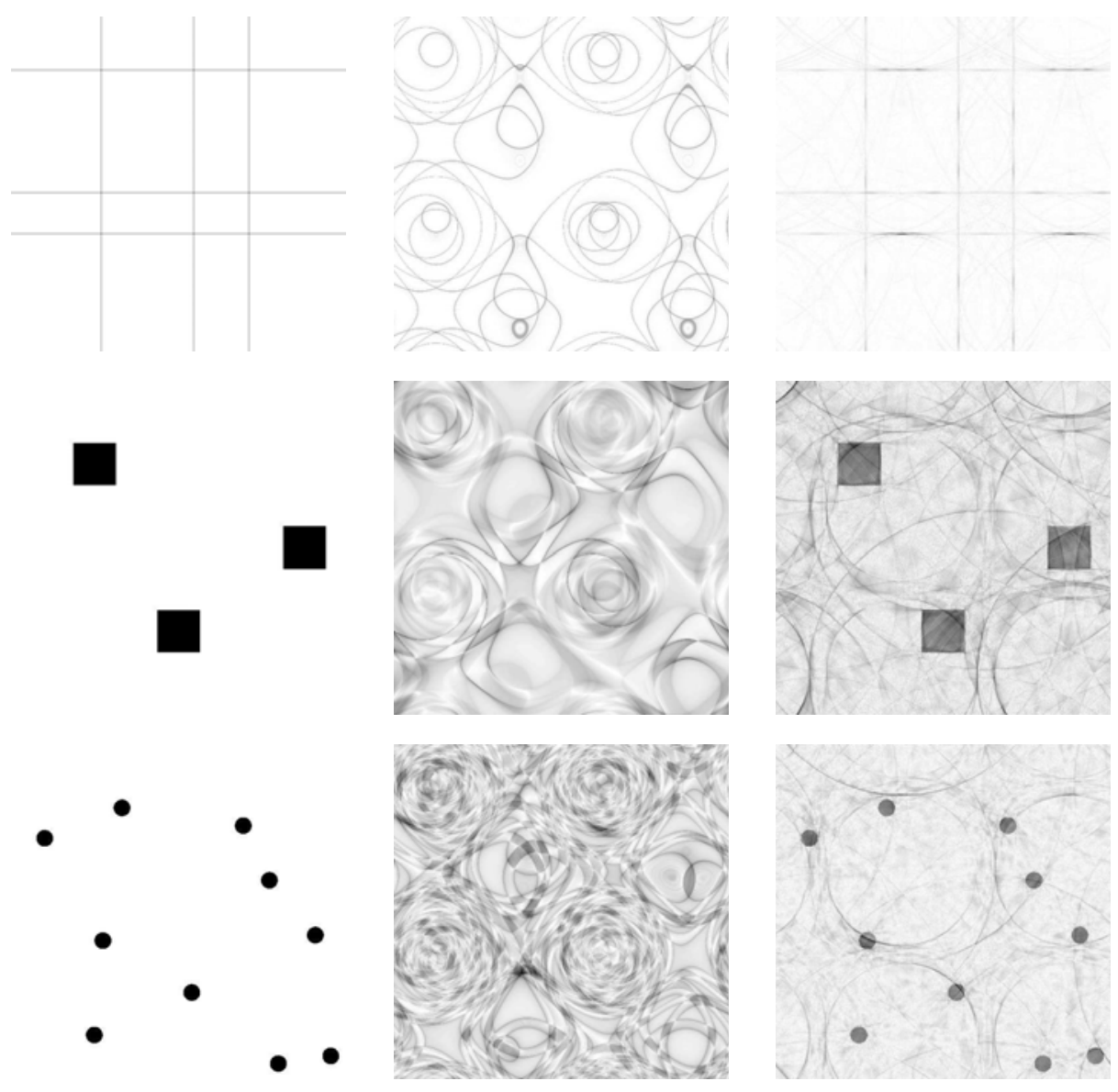

Fig. 2. Numerical verification of the fact $W F\left(L^{*} L f\right)=W F(f)$. Each row, from left to right, shows the magnitudes of $f(x),(L f)(x)$, and $\left(L^{*} L f\right)(x)$. Notice that the wave front set of $f(x)$ and $\left(L^{*} L f\right)(x)$ are numerically close. Remark: The images in the left column and in the right column are not supposed to be the same; only their "singularities" coincide. In other words, the adjoint $L^{*}$ is not the inverse of $L$. The artifacts appear because the data to which we apply the FIO is not bandlimited.

\section{Discussion.}

5.1. About randomized algorithms. The method used in the randomized preprocessing step was first introduced by Kapur and Long [30]. Lately, there has been a lot of research devoted to the development of randomized algorithms for generating low rank factorizations, and we would like to discuss some of this work.

Drineas, Kannan, and Mahoney [19] describe a randomized algorithm for computing a low rank approximation to a fixed matrix. The main idea is to form a submatrix by selecting columns with a probability proportional to their norm. Since this work is about unstructured general matrices, it does not guarantee a small approximation error. As an example, suppose all the columns of the matrix have the same norm and one of them is orthogonal to the span of the other columns. Unless this column is selected, the orthogonal component is lost and the resulting approximation is poor. 
Our situation is different. Since each entry of our matrix

$$
A=\left(e^{2 \pi i \Phi_{\ell}(x, \xi)}\right)_{x \in X, \xi \in W_{\ell}}
$$

has unitary magnitude, the uniform probability used in our algorithm is actually the same as that proposed above [19]. In some ways, then, our approach is a special case of that of Drineas, Kannan, and Mahoney. But the point is that our matrix has a special structure. As we argued earlier, the columns of $A$ are often highly correlated, and we believe that this is the reason why the randomized subsampling performs well.

A recent article by Martinsson, Rokhlin, and Tygert [32] presents a new randomized solution to the same problem. The only inconvenience of this algorithm, probably inevitable for general matrices, is that one needs to visit all the entries of the matrix multiple times. This can be quite costly in our setup since there are $O\left(N^{4}\right)$ entries. This is why we adopt the method by Kapur and Long.

5.2. Storage compression. We would like to comment on the storage compression strategy discussed at the end of section 3.2. In fact, what we described there can be viewed as a way of compressing low rank matrices.

In a general context, the entries of a matrix can be viewed as interaction coefficients between a set of objects indexed by the rows and another set indexed by the columns. In our case, the first set contains the grid points $x$ in $X$, while the second set consists of the frequencies $\xi$ in $W_{\ell}$. Call these two sets $I$ and $J$, and the interaction matrix $A_{I, J}$. The standard practice for compressing $A_{I, J}$ is to find two sets $I^{\prime}$ and $J^{\prime}$ of smaller sizes and form an approximation

$$
A_{I, J} \approx M_{I, I^{\prime}} M_{I^{\prime}, J^{\prime}} M_{J^{\prime}, J} .
$$

Here $I^{\prime}$ is either a subset of $I$ or a set which is close by in some sense, and likewise for $J^{\prime}$ and $J$. For example, in the fast multipole method of Greengard and Rokhlin [26], $J^{\prime}$ is the multipole representation at the center of the box containing $J$, while $I^{\prime}$ is the local representation at the center of the box containing $I$. The matrices $M_{I, I^{\prime}}$, $M_{I^{\prime}, J^{\prime}}$, and $M_{J^{\prime}, J}$ are implemented as the multipole-to-multipole, multipole-to-local, and local-to-local translations. This becomes even more obvious when one considers the newly proposed kernel independent fast multipole method by Ying, Biros, and Zorin [40]. There, $I^{\prime}$ and $J^{\prime}$ are the equivalent densities supported on the boxes containing $I$ and $J$, while $M_{I, I^{\prime}}, M_{I^{\prime}, J^{\prime}}$, and $M_{J^{\prime}, J}$ can be computed directly from interaction matrices and their inverses. In both cases, we are fortunate in the sense that prior knowledge offers us efficient ways to multiply $M_{I, I^{\prime}}, M_{I^{\prime}, J^{\prime}}$, and $M_{J^{\prime}, J}$ with arbitrary vectors. Whenever this is not true, one might be forced to store these matrices, which could be quite costly.

What we have presented in (3.3) is a different factorization:

$$
A_{I J} \approx A_{I J^{\prime}} R_{J^{\prime} I^{\prime}} A_{I^{\prime} J}
$$

This factorization can be viewed as a variant of the pseudoskeleton approximation proposed in $[23,24]$, which came to our attention after we had released the initial version of this paper. Notice that since $A_{I J^{\prime}}$ and $A_{I^{\prime} J}$ are interaction matrices themselves, there is no need to store them as long as we can compute the interaction coefficients easily. The only thing we need to keep in storage is the matrix $R_{J^{\prime} I^{\prime}}$. However, as long as the interaction is low rank, $I^{\prime}$ and $J^{\prime}$ have far fewer objects than $I$ and $J$, so that $R_{J^{\prime} I^{\prime}}$ uses only very little storage. Finally, we would like to point out 


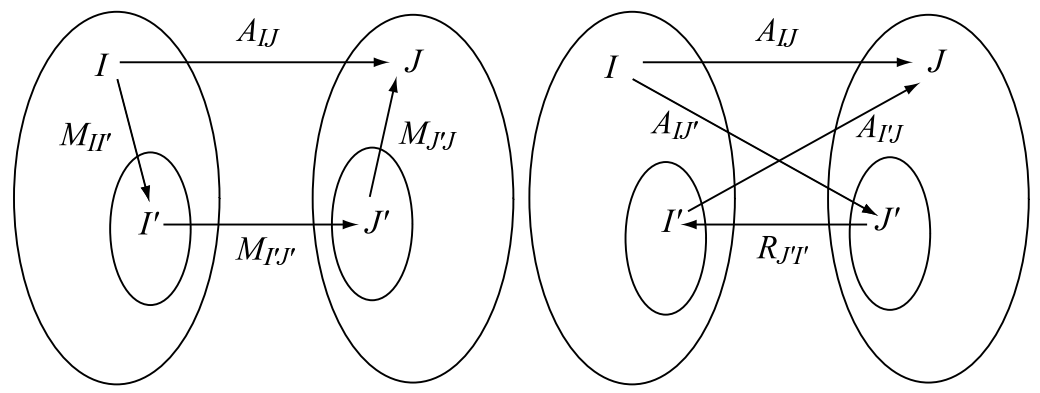

FIG. 3. Factorization of interaction between $A$ and $B$. (a) The standard scheme. (b) The scheme abstracted from the storage compression method used in (3.3).

that, instead of representing the interaction from $J^{\prime}$ (a subset of $J$ ) to $I^{\prime}$ (a subset of $I), R_{J^{\prime} I^{\prime}}$ is a reverse interaction. Figure 3 shows conceptually how the current factorization differs from the standard one.

5.3. Curvelets, wave atoms, and beamlets. There might be other ways of evaluating FIOs, and we would like to discuss their relationships to the approach taken in this paper.

Curvelets, proposed by Candès and Donoho [12], are two-dimensional waveforms which are highly anisotropic in the fine scales. Each curvelet is identified with three numbers to indicate its scale, orientation, and position, and the set of all curvelets forms a tight frame. Recently, Candès and Demanet $[10,11]$ have shown that the curvelet representation of the FIOs is optimally sparse. More precisely, a FIO has only $O\left(N^{2}\right)$ nonnegligible entries in the curvelet domain. The wave atom frame, which was recently introduced by Demanet and Ying [16], has the same property. If we were able to find such a representation efficiently, we would hold an $O\left(N^{2} \log N\right)$ algorithm for evaluating a FIO which would operate as follows:

1. Apply the forward curvelet transform to the input and get curvelet coefficients.

2. Apply the sparse FIO to the curvelet coefficient sequence.

3. Apply the inverse curvelet transform.

Both steps 1 and 3 require at most $O\left(N^{2} \log N\right)$ operations [13].

Constructing the curvelet representation of a FIO from the phase function $\Phi(x, \xi)$ efficiently has, however, proved to be nontrivial. At the moment, we are able only to construct an approximation which is asymptotically accurate by studying the canonical relation embedded inside the phase function $\Phi(x, \xi)$. Such a construction would be adequate if we were interested in applying a FIO to input functions with only high frequency modes. However, one often wants a representation which is accurate for all frequency modes, and we are currently not aware of any efficient method for constructing such a representation.

Beamlets [17] were introduced by Donoho and Huo at roughly the same time as curvelets. Beamlets are small segments at different positions, scales, and orientations. As pointed out in section 4, curvilinear integrals make up an important subclass of FIOs, and beamlets may offer ways to efficiently compute such simpler integrals. One might think of something like this:

1. Compute the beamlet coefficient sequence of the input.

2. For each $x \in X$, figure out the integration curve and approximate it with a chain of beamlet segments. Sum up the beamlet coefficients along the chain. 
Assuming the integration curves are twice differentiable, we would need about $\sqrt{N}$ beamlet segments to approximate each curve. Thus, the overall complexity of this algorithm might scale like $O\left(N^{2.5}\right)$, which is the same scaling as that of our algorithm. The problem is that it is unclear how one would efficiently approximate the integration curve with beamlet segments without sacrificing accuracy. Situations in which the input function $f$ is highly oscillatory or in which the integration curves have parts with a high curvature seem very problematic.

Our algorithms decompose the FIO in the frequency domain, whereas the beamlet based approach processes data in the spatial domain. Sandwiched right in the middle, curvelets and wave atoms operate in the phase-space - the product of the frequency and of the spatial domains. We believe that operating in phase-space by exploiting the microlocal properties of FIOs would be important in bringing down the complexity to the optimal value of about $N^{2}$ operations.

Appendix. Integration along ellipses. The material in this section is probably not new, but we expand on it for the convenience of the nonspecialist. Consider the generalization Radon transform that consists in integrating $f(x)$ along ellipses of axes lengths $r_{1}(x)$ and $r_{2}(x)$, and centered around $x$ :

$$
G f(x)=\int f\left(x+\left(\begin{array}{l}
r_{1}(x) \cos \theta \\
r_{2}(x) \sin \theta
\end{array}\right)\right) d \theta .
$$

We want to recast it as a sum of FIOs. Let us start by writing

$$
G f(x)=\int K(x, \xi) \hat{f}(\xi) d \xi
$$

with

$$
K(x, \xi)=e^{2 \pi i x \cdot \xi} \int \exp \left[2 \pi i\left(\begin{array}{l}
r_{1}(x) \cos \theta \\
r_{2}(x) \sin \theta
\end{array}\right) \cdot \xi\right] d \theta
$$

Put $\rho(x, \xi)=\sqrt{r_{1}^{2}(x) \xi_{1}^{2}+r_{2}^{2}(x) \xi_{2}^{2}}$ and rewrite

$$
K(x, \xi)=e^{2 \pi i x \cdot \xi} \int \exp \left[2 \pi i \rho(x, \xi)\left(\begin{array}{c}
\cos \theta \\
\sin \theta
\end{array}\right) \cdot\left(\begin{array}{c}
\alpha(x, \xi) \\
\beta(x, \xi)
\end{array}\right)\right] d \theta .
$$

Here $\alpha^{2}+\beta^{2}=1$, and both $\alpha$ and $\beta$ depend on $x$ and $\xi$, but the value of the integral is independent of their particular value. This is because any change of variables $\theta \rightarrow \theta+\phi(x, \xi)$, effectively corresponding to a rotation of the unit vector $(\alpha, \beta)$, keeps the integral invariant. So we may as well take $\alpha=1, \beta=0$ and obtain

$$
K(x, \xi)=e^{2 \pi i x \cdot \xi} \int e^{2 \pi i \rho(x, \xi) \cos \theta} d \theta=\frac{e^{2 \pi i x \cdot \xi}}{2 \pi} J_{0}(2 \pi \rho(x, \xi)) .
$$

Of course the Bessel function $J_{0}$ oscillates, and we need to extract the phase from its asymptotic behavior

$$
J_{0}(2 \pi \rho(x, \xi)) \sim \sqrt{\frac{1}{\pi^{2} \rho(x, \xi)}} \cos \left(2 \pi \rho(x, \xi)-\frac{\pi}{4}\right) .
$$

The idea is now to express $J_{0}(2 \pi \rho(x, \xi))$ as a sum of two terms, each of which is the product between a smooth amplitude (a demodulated version of $J_{0}$ or the envelope of 
$J_{0}$ if you will) and the oscillatory exponential $e^{ \pm 2 \pi i \rho(x, \xi)}$. In effect, $K$ is decomposed as a sum of two FIOs:

$$
K(x, \xi)=a_{+}(x, \xi) e^{2 \pi i \Phi_{+}(x, \xi)}+a_{-}(x, \xi) e^{2 \pi i \Phi_{-}(x, \xi)}
$$

with

$$
\Phi_{ \pm}(x, \xi)=x \cdot \xi \pm \rho(x, \xi) .
$$

There are different ways to choose the amplitudes. One way is to let $Y_{0}$ be the Bessel function of the second kind of order zero [1] and exploit the identity $2 J_{0}=$ $\left(J_{0}+i Y_{0}\right)+\left(J_{0}-i Y_{0}\right)$, which allows us to write

$$
a_{ \pm}(x, \xi)=\frac{1}{4 \pi}\left(J_{0}(2 \pi \rho(x, \xi)) \pm i Y_{0}(2 \pi \rho(x, \xi))\right) e^{\mp 2 \pi i \rho(x, \xi)} .
$$

Both amplitudes behave asymptotically like $\sqrt{1 / \pi^{2} \rho(x, \xi)}$ as $x \rightarrow \infty$, which incidentally shows that the order of the FIO is $-1 / 2$. The logarithmic singularity of $Y_{0}$ near the origin in $\xi$ is mild and easily regularized with no loss of accuracy.

Acknowledgments. We are thankful to William Symes for stimulating discussions about Kirchhoff migration and related topics, to Mark Tygert for pointing our attention to the pseudoskeleton approximation, and to the referees for their scrutiny.

\section{REFERENCES}

[1] M. Abramowitz and I. A. Stegun, Handbook of Mathematical Functions with Formulas, Graphs, and Mathematical Tables, Dover, New York, 1972.

[2] C. Anderson and M. D. Dahleh, Rapid computation of the discrete Fourier transform, SIAM J. Sci. Comput., 17 (1996), pp. 913-919.

[3] A. Averbuch, E. Braverman, R. Coifman, M. Israeli, and A. Sidi, Efficient computation of oscillatory integrals via adaptive multiscale local Fourier bases, Appl. Comput. Harmon. Anal., 9 (2000), pp. 19-53.

[4] G. Bao And W. W. Symes, Computation of pseudo-differential operators, SIAM J. Sci. Comput., 17 (1996), pp. 416-429.

[5] G. Beylkin, On the fast Fourier transform of functions with singularities, Appl. Comput. Harmon. Anal., 2 (1995), pp. 363-381.

[6] G. Beylkin, The inversion problem and application of the generalized Radon transform, Comm. Pure Appl. Math., 37 (1984), pp. 579-599.

[7] G. Beylkin, Imaging of discontinuities in the inverse scattering problem by inversion of a causal generalized Radon transform, J. Math. Phys., 26 (1985), pp. 99-108.

[8] G. Beylkin, R. Coifman, And V. Rokhlin, Fast wavelet transforms and numerical algorithms. I, Comm. Pure Appl. Math., 44 (1991), pp. 14-183.

[9] B. Bradie, R. Coifman, and A. Grossman, Fast numerical computation of oscillatory integrals related to acoustic scattering, I, Appl. Comput. Harmon. Anal., 1 (1993), pp. 94-99.

[10] E. J. Candès and L. Demanet, Curvelets and Fourier integral operators, C. R. Math. Acad. Sci. Paris, 336 (2003), pp. 395-398.

[11] E. J. Candès And L. Demanet, The curvelet representation of wave propagators is optimally sparse, Comm. Pure Appl. Math., 58 (2005), pp. 1472-1528.

[12] E. J. CAndès AND D. L. Donoho, New tight frames of curvelets and optimal representations of objects with piecewise $C^{2}$ singularities, Comm. Pure Appl. Math., 57 (2004), pp. 219-266.

[13] E. J. Candès, L. Demanet, D. L. Donoho, and L. Ying, Fast discrete curvelet transforms, Multiscale Model. Simul., 5 (2006), pp. 861-899.

[14] W. Chang, P. Carrion, and G. Beylkin, Wavefront sets of solutions to linearised inverse scattering problems, Inverse Problems, 3 (1987), pp. 683-690.

[15] L. Demanet, Curvelets, Wave Atoms, and Wave Equations, Ph.D. thesis, California Institute of Technology, Pasadena, CA, 2006. 
[16] L. Demanet And L. Ying, Wave Atoms and Sparsity of Oscillatory Patterns, Technical report, California Institute of Technology, Pasadena, CA, 2006. Appl. Comput. Harmon. Anal., to appear.

[17] D. L. Donoho And X. Huo, Beamlets and multiscale image analysis, in Multiscale and Multiresolution Methods, Lect. Notes Comput. Sci. Eng. 20, Springer-Verlag, Berlin, 2002, pp. 149-196.

[18] H. Douma And M. V. DE Hoop, Leading-order seismic imaging using curvelets, in SEG Technical Program Expanded Abstracts, 2006, pp. 2411-2415.

[19] P. Drineas, R. Kannan, and M. W. Mahoney, Fast Monte Carlo algorithms for matrices II: Computing a low-rank approximation to a matrix, SIAM J. Comput., 36 (2006), pp. $158-183$.

[20] J. DuistermaAt, Fourier Integral Operators, Birkhäuser Boston, Boston, 1996.

[21] A. Dutt and V. Rokhlin, Fast Fourier transforms for nonequispaced data, SIAM J. Sci. Comput., 14 (1993), pp. 1368-1393.

[22] C. Fefferman, A note on spherical summation multipliers, Israel J. Math., 15 (1973), pp. $44-52$.

[23] S. A. Goreinov, E. E. Tyrtyshnikov, and N. L. Zamarashin, A theory of pseudoskeleton approximations, Linear Algebra Appl., 261 (1997), pp. 1-21.

[24] S. A. Goreinov, N. L. Zamarashin, and E. E. Tyrtyshnikov, Pseudoskeleton approximations by submatrices of greatest size, Mat. Zametki, 62 (1997), pp. 619-623.

[25] L. Greengard And J.-Y. Lee, Accelerating the nonuniform fast Fourier transform, SIAM Rev., 46 (2004), pp. 443-454.

[26] L. Greengard and V. Rokhlin, A fast algorithm for particle simulations, J. Comput. Phys., 73 (1987), pp. 325-348.

[27] W. Hackbusch, A sparse matrix arithmetic based on $H$-matrices. I. Introduction to $H$-matrices, Computing, 62 (1999), pp. 89-108.

[28] L. Hörmander, The Analysis of Linear Partial Differential Operators, Springer-Verlag, New York, 1985.

[29] A. IsERLES, On the numerical quadrature of highly oscillating integrals I: Fourier transforms, IMA J. Numer. Anal., 24 (2004), pp. 365-391.

[30] S. KAPUR AND D. E. Long, Ies3: A fast integral equation solver for efficient 3-dimensional extraction, in Proceedings of the 1997 IEEE/ACM International Conference on ComputerAided Design, Washington, DC, 1997, pp. 448-455.

[31] M. P. Lamoureux and G. F. Margrave, An introduction to numerical methods of pseudodifferential operators, in Proceedings of the CIME Workshop on Pseudodifferential Operators, Quantization and Signals, Centraro, Italy, 2006.

[32] P.-G. Martinsson, V. Rokhlin, And M. Tygert, A Randomized Algorithm for the Approximation of Matrices, Technical report, Yale University, New Haven, CT, 2006.

[33] D. Miller, M. Oristaglio, And G. Beylkin, A new slant on seismic imaging: Classical migration and integral geometry, Geophysics, 52 (1987), pp. 943-964.

[34] N. NGuyen and Q. H. Liu, The regular Fourier matrices and nonuniform fast Fourier transforms, SIAM J. Sci. Comput., 21 (1999), pp. 283-293.

[35] D. Potts, G. Steidl, and M. Tasche, Fast Fourier transforms for nonequispaced data: A tutorial, in Modern Sampling Theory, Appl. Numer. Harmon. Anal., Birkhäuser Boston, Boston, 2001, pp. 247-270.

[36] A. Seeger, C. D. Sogge, And E. M. Stein, Regularity properties of Fourier integral operators, Ann. of Math (2), 134 (1991), pp. 231-251.

[37] H. Sмiтh, A Hardy space for Fourier integral operators, J. Geom. Anal., 8 (1998), pp. 629-653.

[38] C. Stolk And M. DE Hoop, Microlocal analysis of seismic inverse scattering in anisotropic elastic media, Comm. Pure Appl. Math., 55 (2002), pp. 261-301.

[39] W. Symes. Mathematics of Reflection Seismology, Technical report, Rice University, Houston, TX, 1998, available at http://www.trip.caam.rice.edu/txt/tripinfo/preamble.pdf.

[40] L. Ying, G. BiRos, AND D. ZoRIN, A kernel-independent adaptive fast multipole algorithm in two and three dimensions, J. Comput. Phys., 196 (2004), pp. 591-626.

Copyright (c) by SIAM. Unauthorized reproduction of this article is prohibited. 